Article

\title{
Basic Human Body Dimensions Relate to Alcohol Dependence and Predict Hospital Readmission
}

\author{
Bernd Lenz ${ }^{1, *}{ }^{\oplus}$, Martin G. Köllner ${ }^{2}{ }^{\oplus}$, Christiane Mühle ${ }^{1} \oplus$, Christian Weinland ${ }^{1}$ and \\ Johannes Kornhuber ${ }^{1}$ \\ 1 Department of Psychiatry and Psychotherapy, Friedrich-Alexander University Erlangen-Nürnberg (FAU), \\ Schwabachanlage 6, D-91054 Erlangen, Germany; christiane.muehle@uk-erlangen.de (C.M.); \\ christian.weinland@uk-erlangen.de (C.W.); johannes.kornhuber@uk-erlangen.de (J.K.) \\ 2 Human Motivation and Affective Neuroscience Lab, Department of Psychology, Institute of Psychology, \\ Friedrich-Alexander University Erlangen-Nürnberg (FAU), Nägelsbachstraße 49 b, D-91052 Erlangen, \\ Germany; martin.koellner@fau.de \\ * Correspondence: bernd.lenz@uk-erlangen.de
}

Received: 2 October 2019; Accepted: 12 November 2019; Published: 27 November 2019

check for updates

\begin{abstract}
Alcohol dependence is a severe mental illness and there is a need for more effective preventive and therapeutic strategies. Translational research suggests that intrauterine sex hormone exposure modulates the risk and course of alcohol dependence during adulthood. During development, sex hormones permanently shape sexually dimorphic body dimensions. Thus, these dimensions may provide insight into sex hormone organization. Here, we compared body measurements (absolute, relative to, and residualized on height) between 200 alcohol-dependent in-patients and 240 age-matched healthy control subjects and investigated how these measurements associate with the patients' prospective 12- and 24-month outcome. The results show that alcohol dependence is related to lower absolute, relative, and residualized body measurements for height and weight, head circumference, bitragion head arc, lip-chin distance, hip, thigh, and calf circumference, and foot length and breadth. In male alcohol-dependent in-patients, higher risk, shorter latency, and more alcohol-related readmissions were predicted by higher absolute, relative, and residualized thigh and calf circumferences. The second-to-fourth finger length ratio, a putative proxy for prenatal sex hormone organization, was not convincingly correlated with the body dimensions, suggesting that the results represent pubertal (or later) effects. The study's findings have implications for further research. The body measurements' high accessibility may facilitate the future transition into clinical settings.
\end{abstract}

Keywords: Alcohol Dependence; Relapse Prediction; Body Measurements; Body Dimensions; Organizational Sex Hormone Effects; Anthropometry; Pubertal Hormones

\section{Introduction}

The World Health Organization (WHO) estimated that the harmful use of alcohol accounted for $5.1 \%$ of all deaths and $5.3 \%$ of all disability-adjusted life years during 2016. Worldwide, the highest levels of alcohol consumption per capita were found within the European region [1]. To develop preventive and individualized treatment strategies, we need to improve the knowledge of the mechanisms that underlie alcohol dependence. Easily accessible and clinically relevant biomarkers for both alcohol dependence per se and its prospective course are required.

In 2016, males in all WHO regions were more likely to be current alcohol drinkers and had heavy drinking sessions more often than females. In the European Region, $14.8 \%$ of males and $3.5 \%$ of females suffered from alcohol use disorder. Globally, 2.3 million male deaths compared to 0.7 million female deaths have been attributed to alcohol [1]. Alcohol dependence often runs a chronic course, with higher 
12-month alcohol-related readmission rates following withdrawal in males (57.5\%) compared to females $(41.4 \%)$ [2]. Men are also known to have higher striatal dopamine release after oral alcohol intake than women [3]. These sex differences suggest that sex hormone activities are involved in alcohol dependence. This assumption is supported by studies that associate alcohol dependence and its related phenotypes with genes that are involved in sex hormone signaling, biosynthesis, and degradation (estrogen receptor $1[4,5]$, androgen receptor [6,7], aromatase [8], $5 \alpha$-reductases [9,10]). However, to our knowledge, the cited genetic links still lack replication and only one association originates from a genome-wide association study [4].

Animal and human studies indicate that during key periods such as the prenatal window and puberty, sex hormones organize the development of the brain with lasting behavioral effects and, in parallel, shape the body with lifelong consequences [11-13]. Hence, permanent sex dimorphisms in many human body dimensions are established during certain developmental episodes and thereafter remain stable or at least do not severely change throughout life. Thus, differences in body dimensions between males and females may be used to investigate the associations of organizing prenatal, pubertal, and later sex hormone exposures with psychiatric disorders, which include alcohol dependence.

The second-to-fourth finger length ratio (2D:4D) has been proposed as a proxy for the intrauterine sex hormone exposure, with lower values indicating prenatal hyperandrogenization $[14,15]$ (for a critical review see [16,17]). Lower 2D:4D has been associated with a whole range of impairments such as behavioral problems in childhood [18], video game addiction [19], problematic and pathological internet use during adolescence [20], suicide in adulthood [21,22], and reduced life expectancy [23-25].

Lower 2D:4D has been reported in males and females that have high alcohol consumption defined as "daily" or "daily+" in a large BBC internet study [26] and as "at least two problems in the CAGE test and alcohol consumption of at least a few times each week" in a young university student sample [27]. Our first investigation on the 2D:4D-alcohol dependence relationship revealed lower 2D:4D in both males and females that were diagnosed with alcohol dependence [28]. Three additional studies on this topic have since been published $[25,29,30]$. In support of the initial investigation, our recent meta-analysis confirmed lower 2D:4D in alcohol-dependent males with a medium effect size (Hedge's $\mathrm{g}=-0.552$ ) [31]. Lower 2D:4D has also been shown to predict more alcohol-related 12-month hospital readmissions in alcohol-dependent in-patients [29]. These results suggest that higher androgen load during early intrauterine development increases the risk of alcohol dependence in adulthood and also predicts a worse outcome in affected in-patients. This early sex hormone activity model for alcohol dependence [32] is in agreement with a rodent study that demonstrated that prenatal androgen receptor inhibition reduces alcohol consumption in adult male mice, and that prenatal androgen receptor stimulation increases alcohol consumption in adult female mice [12]. Further support is provided by the fact that the opioid receptor mu 1 may interact with prenatal androgen exposure [12] and 2D:4D [33] to influence alcohol consumption during adulthood.

Another method to investigate prenatal influences of androgens on adult behaviors is the twin testosterone transfer model (TTT). The TTT is based on the assumption that compared to a female twin, a male twin increases the prenatal androgen load of his co-twin in the shared intrauterine environment $[34,35]$. A study using a discovery sample and an independent replication sample has shown that in males, a male co-twin reduces the risk for alcohol dependence during adulthood; in this study, there was no significant effect of the co-twin's sex on risk of alcohol dependence in females [36]. Another study exclusively on females showed more lifetime alcohol use disorder symptoms in women with a male co-twin than in women with a female co-twin [37]. The inconsistent results in females may indicate false positive findings. By contrast, the consistent observations in males suggest that higher androgen load during early intrauterine development might decrease the risk of alcohol dependence in adulthood, which is in line with the early sex hormone activity model of alcohol dependence. However, it also implies a protective effect of higher prenatal androgen exposure in males, which may seem contradictory to the 2D:4D results described above. 2D:4D and the TTT may provide insight regarding the different prenatal developmental windows. 2D:4D sex dimorphisms are thought to originate 
during early pregnancy (i.e., before gestational week 9 [38] and 14 [39]). Thus, 2D:4D is believed to represent a marker of the first trimester. Because androgen levels in male fetuses peak between gestational weeks 12 and 18, the TTT may be used to investigate phenotypes that are established during the second trimester [35]. However, other explanations might also account for the inconsistent findings of studies using 2D:4D and those based on the TTT. Opposite- and same-sex human twins may differ in postnatal social factors (e.g., peer group characteristics) which also influence the risk to develop alcohol dependence later in life. Such bias may entail misinterpretation of results based on the TTT. Additionally, it cannot be ruled out that some of the above cited investigations have produced false positive findings.

In summary, a direct preclinical study and human investigations using the indirect biomarker 2D:4D and the TTT suggest that sex hormone exposure during development modulates the risk of alcohol dependence during adulthood. The organizational effects of sex hormones may depend on the developmental window during which they occur. Although an initial clinical study is being conducted to transfer this knowledge into prevention techniques [40], the field is subject to several limitations that include weaknesses related to 2D:4D and the TTT. Today, we are far from using such knowledge to improve the treatment of alcohol-dependent patients.

Moreover, little is known about the organizational effects of sex hormone exposures during puberty (and later) on alcohol dependence during adulthood. Only recently, the novel research area on sex hormone organization during the pubertal window has evolved [13]. Pubertal sex hormones shape sexual dimorphisms in physical traits and these might, therefore, be used to enlighten the associations between behavioral traits and pubertal sex hormone exposures; e.g., meta-analyses have demonstrated that the facial width-to-height ratio, which is subject to a sexual dimorphism appearing during puberty [41], is related to aggression and threat behavior among males and to dominance behavior in both sexes [42,43].

As far as we know, the association of easily measurable and thus highly accessible sexually dimorphic body dimensions with alcohol dependence and the prospective course of affected in-patients has not been systematically investigated.

\section{Aims of the Study}

Sex dimorphisms in body dimensions can provide initial insights into the organizational effects of sex hormones on the brain with clinically relevant behavioral outcomes. Thus, we analyzed here the differences in 15 absolute body measurements, 13 body measurements divided by height (= relative), and 13 body measurements residualized on height between alcohol-dependent in-patients and healthy control subjects of both sexes. We also investigated their relationships to the patients' prospective 12and 24-month outcome, following alcohol withdrawal treatment, in an effort to identify parameters that will be helpful to determine the individual prognosis. Finally, we explored the associations between absolute, relative, and residualized body measurements with $2 \mathrm{D}: 4 \mathrm{D}$, the age of onset of regular alcohol drinking, the age of the first in-patient treatment due to alcohol problems, total lifetime drinking, and daily ethanol intake.

\section{Materials and Methods}

\subsection{Study Sample}

This project was part of the bicentric, cross-sectional, and prospective Neurobiology of Alcoholism (NOAH) study, which included 200 alcohol-dependent in-patients and 240 age-matched healthy controls subjects [29]. All in-patients were diagnosed with alcohol dependence according to the tenth revision of the International Classification of Diseases (ICD-10) criteria [44] and alcohol use disorder according to the fifth edition of the Diagnostic and Statistical Manual of Mental Disorders (DSM-5) criteria [45]. During the screening procedure, alcohol-dependent in-patients were excluded due to denial of written informed consent $(n=379)$, a mental comorbidity, such as primary depression (i.e., 
depression was present before the alcohol dependence), anxiety disorder, schizophrenia, substance use disorder other than alcohol or nicotine, posttraumatic stress disorder, or eating disorder $(n=194)$, a severe somatic disorder $(n=90)$, abstinence longer than 72 hours prior to inclusion $(n=88)$, or other reasons $(n=37)$. Healthy control subjects were hired with online advertisement and distribution of flyers. After a multistep screening procedure of 1215 interested individuals, 240 healthy control subjects were enrolled. The exclusion criteria of the control group included severe somatic disorders, prior medical treatment due to alcohol dependence, $\geq 2$ affirmed CAGE questions [46], prior psychiatric in-patient treatment, psychiatric or psychotherapeutic outpatient treatment during the past 10 years, and an Alcohol Use Disorders Identification Test (German version [47]) score > 12 .

The NOAH study design included two study-visits for alcohol-dependent in-patients and one study-visit for healthy control subjects. For in-patients, the first study-visit took place during early abstinence (i.e., 24-72 $\mathrm{h}$ of abstinence according to the in-patients' self-reports of their last alcohol intake prior to study inclusion); $81.5 \%$ of the in-patients attended the second direct study-visit, which took place during the median 5th day post-inclusion (interquartile range (IQR) 3-6). The subjects were recruited at the Psychiatrische und Psychotherapeutische Klinik of the Universitätsklinikum Erlangen and at the Klinik für Psychiatrie, Sucht, Psychotherapie und Psychosomatik of the Klinikum am Europakanal, Germany. Post-inclusion, we followed the patients' records for 12 and 24 months to analyze the outcome parameters: alcohol-related readmissions per se, latency (in days) to the first alcohol-related readmission, and total number of alcohol-related readmissions during the 12- and 24-month follow-up periods. For statistical analyses, days to first readmission were set to 365 (12-month follow-up) and 730 days (24-month follow-up) in patients without any recorded alcohol-related readmission during the observation period. Clinically experienced psychiatrists and well-trained and regularly supervised doctoral students (from our study team) conducted the semi-structured interviews. The interviews at study enrolment included the age of onset of regular alcohol drinking (i.e., daily over at least 7 days), the age of first in-patient treatment due to alcohol problems, the alcohol lifetime drinking history [48], and the number of previous in-patient withdrawal treatments in the group of alcohol-dependent in-patients, the Alcohol Use Disorders Identification Test (German version [47]) for the group of healthy control subjects, and smoking status for both groups. We also quantified the breath alcohol concentration in alcohol-dependent in-patients. Moreover, carbohydrate-deficient transferrin (CDT) was measured in serum samples of both groups (Central Laboratory of the University Hospital Erlangen, Germany, DIN EN ISO 15189 accredited). For previously published articles of the NOAH study, see $[2,5,29,33,49-52]$.

\subsection{Ethical Approval}

This study was approved by the Ethics Committee of the Medical Faculty from the Friedrich-Alexander University Erlangen-Nürnberg (ID 81_12 B; April 19, 2012). All participants provided written informed consent. The study is in accordance with the ethical principles of the World Medical Association (sixth revision of the Declaration of Helsinki, Seoul 2008).

\subsection{Basic Human Body Dimensions}

We selected body height, body weight, and mandibular arc as established, sexually dimorphic features [53-55]. The lip-chin distance was included as an indicator for jaw height, which is a constituent part of facial masculinity [56]. We used lip-chin distance instead of full jaw height to exclude lower lip height, which is larger in females than males [57]. The waist and hip circumferences were investigated as sexually dimorphic traits that are largely determined during puberty [58]. Additional parameters with likely relationships to skeletal and/or soft-tissue growth in general were included, such as head, thigh, calf, and ankle circumference, bitragion and sagittal head arcs, foot length and breadth.

For the measurements, the participants wore only underwear and took off their shoes. Body height, body weight, head circumference, bitragion and sagittal head arcs, wrist, waist, hip, thigh, and calf circumferences, as well as foot length and breadth were quantified according to the Basic 
human measurements for technological design-Part 1: Body measurement definitions and landmarks (ISO 7250-1:2008); German version EN ISO 7250-1:2010. For body dimensions not included in the above list, we used the following definitions: Mandibular arc, distance between the two anguli mandibulae spanning over the point of the chin; lip-chin distance, lower lip mucocutaneous boundary to platysma mandible insertion; hip circumference, length over the widest part of the gluteal region; ankle circumference, maximum length parallel to the floor over the malleoli mediales and laterales. Body weight was measured in $\mathrm{kg}$ using a weighing scale and length dimensions in $\mathrm{cm}$ with a tape measure. All measurements were conducted once, by a non-blinded rater, in the standing position with the exception of foot length and breadth. Soles of the right and left feet were scanned in the sitting position using an HP Scanjet G4050 (HP Deutschland GmbH, Böblingen, Germany). Foot length and breadth were quantified by three independent and blinded raters with Microsoft PowerPoint (two-way random intra-class correlation coefficients (absolute agreement): mean of right and left foot length 0.996 , mean of right and left foot breadth 0.990 ). We did not exclude hallux valgus deformations. Most of the missing values in the alcohol-dependent in-patients were due to a lower participation rate in the second study-visit, during which body measurements were conducted (if not already done during the first study visit).

\section{4. $2 D: 4 D$}

Right and left hands were scanned using an HP Scanjet G4050. The absolute index and ring finger lengths were quantified by three independent blinded raters and we analyzed the means of the right-hand and left-hand 2D:4D values, which are published (GNU Image Manipulation Program, www.gimp.org, two-way random intra-class correlation coefficient (absolute agreement) of mean of the right-hand and left-hand 2D:4D 0.986) [29].

\subsection{Statistical Analyses}

Data were analyzed using SPSS for Windows 24.0 (SPSS Inc., Chicago, IL, USA). The descriptive statistics report medians, IQR, and frequencies, which were calculated with the custom tables function of SPSS. We used the Mann-Whitney $U$ test and Spearman's correlation because some of the body measurements deviated significantly from a normal distribution, according to the Kolmogorov-Smirnov test. The $\chi^{2}$ test was employed to analyze differences in the frequency of nominal variables. Values of $P<0.05$ for two-tailed tests were considered significant. To respect the well-known sex differences in alcohol dependence [32], we analyzed males and females separately.

Mean values of right and left-side body measurements were analyzed for wrist, thigh, calf, and ankle circumferences as well as foot length and breadth. We tested for associations of absolute body measurements, body measurements divided by height, and body measurements residualized on height with alcohol dependence and outcome. Body measurements were divided by height to obtain a relative measure in analogy to those frequently used in other fields of marker research (e.g., 2D:4D, facial width-to-height ratio). Moreover, the absolute values of body measurements were residualized for height to determine possible sex differences in and the predictive value of each parameter above and beyond general differences in body height. Linear regression, using the whole study population, was applied to residualize each single body measurement (dependent variable) on body height (predictor). The obtained residuals were interpreted as body measurement values that are independent of general body height. Residuals were z-standardized.

The field of organizational sex hormone effects in alcohol dependence is early in development. Hence, we tested a high number of predictors. In total, we predefined the following 656 statistical tests:

- 82 group comparisons "alcohol-dependent in-patients versus healthy control subjects": ( 15 absolute body measurements +13 body measurements divided by height +13 body measurements residualized on height) $\times 2$ sexes 
- 164 group comparisons "readmission yes versus no": (15 absolute body measurements +13 body measurements divided by height +13 body measurements residualized on height) $\times 2$ sexes $\times 2$ follow-up periods (12-month, 24-month)

- 328 correlations with "outcome parameters": (15 absolute body measurements +13 body measurements divided by height +13 body measurements residualized on height $) \times 2$ sexes $\times 2$ follow-up periods (12-month, 24-month) $\times 2$ outcome parameters (latency, number)

- 82 group comparisons "male versus female": (15 absolute body measurements +13 body measurements divided by height +13 body measurements residualized on height $) \times 2$ groups (alcohol-dependent in-patients versus healthy control subjects)

All reported $P$ values are uncorrected. To consider type 1 error risk in multiple hypothesis testing, we employed the false discovery rate (FDR) procedure using a macro for Microsoft Excel (see Appendix S1 of Pike [59], classical one-stage method including all 656 tests; critical $P$ value (FDR-derived significance threshold): 0.007470; [60]). In parallel, we also employed the more conservative Bonferroni adjustment (critical $P$ value: $0.05 / 656=0.000076$ ). We chose both correction methods to differentiate between robust (FDR) and very robust (Bonferroni) results. However, we acknowledge the problem of statistical power in view of the high number of tests. The sample size has been initially chosen to demonstrate group differences in 2D:4D already published in [29]. The body measurements analyzed in this article represent secondary endpoints and were not included into the a priori sample size estimation.

Finally, we performed the following explorative analyses that were not corrected for multiple hypothesis testing:

- Correlations of absolute, relative, and residualized body measurements with 2D:4D in alcohol-dependent in-patients and healthy control subjects of both sexes.

- Correlations of absolute, relative, and residualized body measurements with the age of onset of regular alcohol drinking and the age of first in-patient treatment due to alcohol problems in alcohol-dependent in-patients of both sexes.

- Correlations of absolute, relative, and residualized body measurements with total lifetime drinking and daily ethanol intake in alcohol-dependent in-patients of both sexes.

- Inter-correlations among the body measurements within the three groups of absolute, relative, and residualized body dimensions in alcohol-dependent in-patients and healthy control subjects of both sexes.

\section{Results}

\subsection{Sociodemographic Characteristics}

Both male and female alcohol-dependent in-patients did not significantly differ from healthy control subjects with regard to age, but were more often active and ever-smokers and had higher CDT blood levels. In the group of alcohol-dependent in-patients, male sex was significantly related to a younger age of onset of regular alcohol drinking (i.e., daily over at least 7 days), higher total lifetime drinking and daily ethanol intake since onset, a higher alcohol concentration at admission, higher CDT blood levels, and worse 12- and 24-month outcomes. Moreover, we found significantly higher Alcohol Use Disorders Identification Test scores and higher CDT blood levels in male (than in female) healthy control subjects (Table 1). 
Table 1. Demographic characteristics of male and female alcohol-dependent in-patients (ADP) and healthy control subjects (HCS).

\begin{tabular}{|c|c|c|c|c|c|c|c|c|c|c|c|c|c|c|c|c|c|c|c|c|c|c|c|c|}
\hline & \multicolumn{8}{|c|}{ Males } & \multicolumn{8}{|c|}{ Females } & \multicolumn{4}{|c|}{ ADP versus HCS } & \multicolumn{4}{|c|}{ Males versus Females } \\
\hline & \multicolumn{4}{|c|}{$\operatorname{ADP}(N=113)$} & \multicolumn{4}{|c|}{ HCS $(N=133)$} & \multicolumn{4}{|c|}{ ADP $(N=87)$} & \multicolumn{4}{|c|}{ HCS $(N=107)$} & \multicolumn{2}{|c|}{ Males } & \multicolumn{2}{|c|}{ Females } & \multicolumn{2}{|c|}{ ADP } & \multicolumn{2}{|c|}{ HCS } \\
\hline & $N$ & $\mathrm{M} / \mathrm{F}$ & \multicolumn{2}{|c|}{ IQR } & \multirow{2}{*}{$\begin{array}{c}N \\
133\end{array}$} & \multirow{2}{*}{$\begin{array}{l}\mathrm{M} / \mathbf{F} \\
48\end{array}$} & \multicolumn{2}{|c|}{ IQR } & \multirow{2}{*}{$\begin{array}{l}N \\
87\end{array}$} & \multirow{2}{*}{$\begin{array}{c}\mathrm{M} / \mathrm{F} \\
48\end{array}$} & \multicolumn{2}{|c|}{ IQR } & \multirow{2}{*}{$\begin{array}{c}N \\
107\end{array}$} & \multirow{2}{*}{$\begin{array}{l}\mathrm{M} / \mathrm{F} \\
49\end{array}$} & \multicolumn{2}{|c|}{ IQR } & \multirow{2}{*}{$\begin{array}{l}U / x^{2} \\
7369\end{array}$} & \multirow{2}{*}{$\begin{array}{c}P \\
0.794\end{array}$} & \multirow{2}{*}{$\frac{U / x^{2}}{4542}$} & \multirow{2}{*}{\begin{tabular}{|c|}
$P$ \\
0.772
\end{tabular}} & $u / x^{2}$ & $P$ & $u / x^{2}$ & $P$ \\
\hline Age (years) & 113 & 48 & 40 & 53 & & & 38 & 56 & & & 42 & 55 & & & 39 & 55 & & & & & 4502 & 0.308 & 6954 & 0.762 \\
\hline $\begin{array}{l}\text { The age of onset of regular } \\
\text { alcohol drinking } \\
\text { (i.e., daily over at least } 7 \text { days) } \\
\text { (years) }\end{array}$ & 92 & 26 & 19 & 34 & & - & & & 67 & 33 & 23 & 41 & & - & & & & & & & 2152 & 0.001 & & \\
\hline $\begin{array}{l}\text { The age of first in-patient } \\
\text { treatment due to alcohol } \\
\text { problems (years) }\end{array}$ & 96 & 36 & 30 & 47 & & - & & & 77 & 40 & 32 & 46 & & - & & & & & & & 3256 & 0.179 & & \\
\hline Total lifetime drinking $(\mathrm{kg})$ & 88 & 705 & 364 & 1838 & & - & & & 62 & 332 & 174 & 747 & & - & & & & & & & 1485 & $<0.001$ & & \\
\hline $\begin{array}{l}\text { Previous withdrawal } \\
\text { treatments }(n)\end{array}$ & 89 & 6 & 2 & 12 & & - & & & 58 & 5 & 2 & 11 & & - & & & & & & & 2547 & 0.892 & & \\
\hline $\begin{array}{l}\text { Alcohol concentration at } \\
\text { admission }(\%)\end{array}$ & 108 & 1.7 & 0.5 & 2.4 & & - & & & 85 & 1.2 & 0.1 & 1.8 & & - & & & & & & & 3696 & 0.020 & & \\
\hline AUDIT score & & - & & & 125 & 4 & 3 & 6 & & - & & & 96 & 3 & 2 & 4 & & & & & & & 4295 & $<0.001$ \\
\hline Active smokers (\%) & 104 & 78 & & & 133 & 22 & & & 78 & 77 & & & 107 & 19 & & & 74 & $<0.001$ & 62 & $<0.001$ & $<1$ & 0.878 & $<1$ & 0.552 \\
\hline Active and ex-smokers (\%) & 101 & 92 & & & 133 & 59 & & & 75 & 85 & & & 105 & 48 & & & 31 & $<0.001$ & 27 & $<0.001$ & 2 & 0.154 & 3 & 0.070 \\
\hline CDT (nephelometry, \%) & 113 & 2.8 & 1.9 & 4.0 & 132 & 1.5 & 1.3 & 1.7 & 87 & 1.9 & 1.6 & 2.5 & 107 & 1.5 & 1.3 & 1.6 & 1636 & $<0.001$ & 1415 & $<0.001$ & 3003 & $<0.001$ & 6692 & 0.486 \\
\hline 12-month alcohol-related readmis & sions & & & & & & & & & & & & & & & & & & & & & & & \\
\hline Readmission rate (\%) & 113 & 58 & & & & - & & & 87 & 41 & & & & - & & & & & & & 5 & 0.024 & & \\
\hline 24-month alcohol-related readmis & sions & & & & & & & & & & & & & & & & & & & & & & & \\
\hline Readmission rate $(\%)$ & 113 & 67 & & & & - & & & 87 & 53 & & & & - & & & & & & & 4 & 0.039 & & \\
\hline Latency (days) & 113 & 285 & 57 & $\geq 730$ & & - & & & 87 & 625 & 90 & $\geq 730$ & & - & & & & & & & 4136 & 0.047 & & \\
\hline Total number & 113 & 2 & 0 & 4 & & - & & & 87 & 1 & 0 & 3 & & - & & & & & & & 4014 & 0.021 & & \\
\hline
\end{tabular}

The table shows medians $(\mathrm{M})$ or relative frequencies $(\mathrm{F})$, interquartile ranges (IQR), and results of Mann-Whitney $U$ tests or $\chi^{2}$ tests. AUDIT: Alcohol Use Disorders Identification Test; CDT: carbohydrate-deficient transferrin. $P<0.05$ in bold. 


\subsection{Basic Human Body Dimensions: Alcohol-Dependent In-Patients versus Healthy Control Subjects}

After FDR correction, several absolute, relative, and residualized body measurements were significantly and consistently lower in alcohol-dependent in-patients than in healthy control subjects, except for sagittal head arc divided by height, which was significantly longer in alcohol-dependent in-patients (Table 2).

Absolute Body Measurements: Alcohol dependence was related to shorter head, hip, and thigh circumference in both sexes and to lower body height and weight, bitragion head arc length, lip-chin distance, calf circumference, and foot length and breadth in males. Body Measurements Divided by Height: Alcohol dependence was associated with shorter thigh circumference in both sexes, with longer sagittal head arc length and shorter hip and calf circumference in males, and with shorter head circumference in females. Body Measurements Residualized on Height: Alcohol dependence was related to shorter hip and thigh circumferences in both sexes, to a shorter bitragion head arc, calf circumference, and foot breadth in males, and to shorter head circumference in females.

The smaller absolute, relative, and residualized hip, thigh, and calf circumferences and the smaller absolute body weight and foot breadth in male alcohol-dependent in-patients, in comparison to male healthy control subjects, remained significant after the Bonferroni correction.

\subsection{Basic Human Body Dimensions: Prospective Alcohol-Related Readmission}

After FDR correction, worse patients' outcomes were consistently related to significantly higher absolute, relative, and residualized thigh and calf circumferences in male alcohol-dependent in-patients. We did not find any significant association of absolute, relative, or residualized body measurement with outcomes in female alcohol-dependent in-patients (Tables 3-5).

Absolute Body Measurements: Thigh circumference was linked to risk, latency, and the number of alcohol-related readmissions throughout the 12-month follow-up. Calf circumference correlated with the 12-month latency to alcohol-related readmission. Body Measurements Divided by Height: Thigh circumference was associated with risk, latency, and the number of alcohol-related readmissions for the 12-month and 24-month follow-ups. Calf circumference was linked to 12-month risk and 12-month and 24-month latency to alcohol-related readmission. Moreover, there was an association between head circumference values and number of alcohol-related readmissions during the 24-month follow-up. Body Measurements Residualized on Height: Thigh circumference was linked to risk, latency, and the number of alcohol-related readmission for the 12-month follow-up and the number of alcohol-related readmissions for the 24-month follow-up. Calf circumference related to 12-month und 24-month latency to alcohol-related readmission.

None of these effects remained significant after the Bonferroni adjustment.

\subsection{Basic Human Body Dimensions: Sex Differences}

After FDR correction, we found significantly longer absolute and residualized body measurements in males than in females, except for the shorter residualized thigh circumference in male than in female alcohol-dependent in-patients. Many relative body measurements were significantly lower in males than in females (Table 2). 
Table 2. Differences in body measurements between alcohol-dependent in-patients (ADP) and healthy control subjects (HCS) and between males and females (Mann-Whitney $U$ tests).

\begin{tabular}{|c|c|c|c|c|c|c|c|c|c|c|c|c|c|c|c|c|c|c|c|c|c|c|c|c|}
\hline & \multicolumn{8}{|c|}{ Males } & \multicolumn{8}{|c|}{ Females } & \multicolumn{4}{|c|}{ ADP versus HCS } & \multicolumn{4}{|c|}{ Males versus Females } \\
\hline & \multicolumn{4}{|c|}{ ADP } & \multicolumn{4}{|c|}{ HCS } & \multicolumn{4}{|c|}{ ADP } & \multicolumn{4}{|c|}{ HCS } & \multicolumn{2}{|c|}{ Males } & \multicolumn{2}{|c|}{ Females } & \multicolumn{2}{|c|}{ ADP } & \multicolumn{2}{|c|}{ HCS } \\
\hline & $N$ & M & & $2 R$ & $N$ & $\mathbf{M}$ & & $2 R$ & $N$ & M & & $2 R$ & $N$ & M & & & $u$ & $P$ & $u$ & $P$ & $u$ & $P$ & $u$ & $P$ \\
\hline \multicolumn{25}{|l|}{ Absolute measure } \\
\hline Body height & 107 & 176.8 & 172.0 & 181.0 & 133 & 179.1 & 173.8 & 184.1 & 77 & 164.5 & 162.0 & 169.0 & 107 & 165.6 & 161.5 & 169.1 & 5668 & 0.007 & 4064 & 0.876 & 943 & $<0.001 *$ & 864 & $<0.001^{*}$ \\
\hline Head circumference & 107 & 57.5 & 56.2 & 58.9 & 133 & 58.4 & 57.2 & 59.4 & 79 & 55.0 & 54.0 & 56.5 & 107 & 56.0 & 54.5 & 57.3 & 5436 & 0.002 & 3198 & 0.005 & 1348 & $<0.001 *$ & 2626 & $<0.001$ * \\
\hline Bitragion head arc & 107 & 36.7 & 35.6 & 37.5 & 133 & 37.4 & 36.5 & 38.7 & 79 & 35.5 & 34.5 & 36.3 & 107 & 36.0 & 35.0 & 37.4 & 5149 & $<0.001$ & 3318 & 0.012 & 2444 & $<0.001 *$ & 4149 & $<0.001$ * \\
\hline Sagittal head arc & 107 & 36.0 & 35.0 & 37.7 & 133 & 35.6 & 34.5 & 37.0 & 79 & 35.0 & 34.0 & 36.1 & 107 & 34.5 & 33.0 & 36.0 & 6319 & 0.136 & 3900 & 0.367 & 2814 & $<0.001$ & 5030 & $<0.001$ \\
\hline Mandibular arc & 106 & 24.0 & 23.0 & 25.2 & 133 & 24.0 & 22.5 & 26.0 & 77 & 22.2 & 21.3 & 23.0 & 106 & 22.4 & 21.0 & 23.6 & 6905 & 0.785 & 3907 & 0.621 & 1501 & $<0.001 *$ & 3694 & $<0.001 *$ \\
\hline Lip-chin distance & 106 & 5.5 & 4.9 & 6.0 & 132 & 5.6 & 5.4 & 6.0 & 79 & 5.0 & 4.4 & 5.5 & 107 & 5.0 & 4.7 & 5.5 & 5303 & 0.001 & 3446 & 0.031 & 2731 & $<0.001 *$ & 3758 & $<0.001 *$ \\
\hline Wrist circumference & 102 & 17.7 & 16.8 & 18.4 & 128 & 17.9 & 17.2 & 18.6 & 75 & 16.0 & 15.5 & 16.8 & 103 & 15.8 & 14.9 & 16.7 & 5761 & 0.126 & 3382 & 0.156 & 1037 & $<0.001 *$ & 1825 & $<0.001 *$ \\
\hline Hip circumference & 106 & 94.2 & 88.8 & 100.0 & 133 & 102.0 & 98.0 & 107.0 & 77 & 96.0 & 90.8 & 106.0 & 107 & 101.2 & 95.0 & 106.8 & 3076 & $<0.001$ * & 3130 & 0.005 & 3424 & 0.063 & 6342 & 0.148 \\
\hline Thigh circumference & 106 & 55.2 & 50.8 & 58.5 & 133 & 60.5 & 57.8 & 64.6 & 79 & 56 & 53.7 & 62.0 & 106 & 60. & 57.0 & 64.0 & 3043 & $<0.001$ * & 3000 & 0.0 & 32 & 0.0 & & 0.426 \\
\hline Calf circumference & 106 & 36.8 & 35.4 & 38.9 & 132 & 39.5 & 37.7 & 41.5 & 79 & 36.0 & 33.8 & 39.2 & 107 & 37.3 & 35.0 & 39.5 & 3785 & $<0.001 *$ & 3647 & 0.110 & 3657 & 0.141 & 3999 & $<0.001 *$ \\
\hline Ankle circumference & 106 & 26.2 & 25.3 & 27.2 & 131 & 26.6 & 25.9 & 27.9 & 79 & 24.2 & 23.1 & 25.8 & 107 & 24.6 & 23.6 & 25.4 & 5720 & 0.020 & 4107 & 0.741 & 1922 & $<0.001 *$ & 2313 & $<0.001 *$ \\
\hline Foot length & 99 & 26.1 & 25.1 & 27.0 & 105 & 26.9 & 26.0 & 27.6 & 77 & 24.0 & 23.1 & 24.7 & 100 & 24.1 & 23.5 & 25.0 & 3625 & $<0.001$ & 3413 & 0.195 & 805 & $<0.001 *$ & 733 & $<0.001 *$ \\
\hline Foot breadth & 100 & 9.7 & 9.4 & 10.1 & 107 & 10.1 & 9.8 & 10.4 & 78 & 9.1 & 8.8 & 9.5 & 101 & 9.2 & 8.9 & 9.4 & 3375 & $<0.001$ * & 3930 & 0.978 & 1559 & $<0.001 *$ & 725 & $<0.001 *$ \\
\hline Measure divided by $b$ & dy he & & & & & & & & & & & & & & & & & & & & & & & \\
\hline Head circumference & 107 & 0.33 & 0.32 & 0.33 & 133 & 0.32 & 0.32 & 0.33 & 77 & 0.33 & 0.32 & 0.34 & 107 & 0.34 & 0.33 & 0.35 & 6846 & 0.614 & 3142 & 0.006 & 3036 & 0.002 & 3505 & \\
\hline Bitragion head arc & 107 & 0.21 & 0.20 & 0.21 & 133 & 0.21 & 0.20 & 0.22 & 77 & 0.22 & 0.21 & 0.22 & 107 & 0.22 & 0.21 & 0.23 & 6394 & 0.177 & 3251 & 0.015 & 2492 & $<0.001 *$ & 3940 & $<0.001 *$ \\
\hline Sagittal head arc & 107 & 0.21 & 0.20 & 0.21 & 133 & 0.20 & 0.19 & 0.21 & 77 & 0.21 & 0.20 & 0.22 & 107 & 0.21 & 0.20 & & 5313 & 0.001 & 3933 & 0.601 & & & & \\
\hline & 106 & 0.14 & 0. & & & 0.14 & 0.13 & & 75 & & 0.13 & & 10 & 0.1 & 0.13 & & 66 & 0.4 & 37 & 0. & 3551 & 0.222 & 6906 & 0.788 \\
\hline Lip-chin distar & 106 & 0.03 & 0.03 & 0.03 & 132 & 0.03 & 0.03 & 0.03 & 77 & 0.0 & 0.03 & 0.03 & 107 & 0.0 & 0.03 & & 5843 & 0.0 & 3299 & 0.0 & 3634 & 0.206 & 6382 & 0.200 \\
\hline Wrist circumference & 102 & 0.10 & 0.10 & 0.10 & 128 & 0.10 & 0.10 & 0.10 & 73 & 0.10 & 0.09 & 0.10 & 103 & 0.10 & 0.09 & 0.10 & 6366 & 0.746 & 3343 & 0.211 & 2761 & 0.004 & 4722 & $<0.001$ \\
\hline Thigh c & 106 & 0.31 & 0.2 & 0. & 133 & 0.34 & 0.32 & & 77 & & 0.33 & & 10 & 0. & 0.34 & & 3603 & $<0.00$ & 2976 & & & & 4234 & \\
\hline Calf cir & 106 & 0.21 & 0. & 0. & 1 & 0. & 0. & & 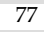 & & 0.21 & & 10 & 0. & 0. & & 47 & $<0.001 *$ & 34 & 0.0 & 3078 & 0.005 & 6078 & 0.064 \\
\hline Ankle circumference & 106 & 0.15 & 0.14 & 0.15 & 131 & 0.15 & 0.1 & 0. & 77 & 0. & 0.14 & 0.1 & 107 & 0.15 & 0.14 & 0.16 & 6535 & 0.437 & 3943 & 0.61 & 4036 & 0.898 & 6769 & 0.650 \\
\hline Foot length & 99 & 0.15 & 0.14 & 0.15 & 105 & 0.15 & 0.15 & 0.15 & 74 & 0.14 & 0.14 & 0.15 & 100 & 0.15 & 0.14 & 0.15 & 4526 & 0.1 & 2843 & 0.009 & 2525 & $<0.001$ & 4158 & 0.010 \\
\hline Foot breadth & 100 & 0.06 & 0.05 & 0.06 & 107 & 0.06 & 0.05 & 0.06 & 75 & 0.06 & 0.05 & 0.06 & 101 & 0.06 & 0.05 & 0.06 & 4296 & 0.014 & 3759 & 0.932 & 3629 & 0.715 & 4594 & 0.062 \\
\hline Measure residualized & on bod & heigh & & & & & & & & & & & & & & & & & & & & & & \\
\hline Head circumference & 107 & 0.08 & -0.46 & 0.66 & 133 & 0.26 & -0.19 & 0.73 & 77 & -0.46 & -1.00 & & 107 & -0.15 & -0.53 & 0.60 & 6241 & 0.102 & 2976 & 0.001 & 2674 & $<0.001$ * & 5615 & 0.005 \\
\hline Bitragi & 107 & -0.09 & -0.75 & 0. & 133 & 0.25 & -0 & & 77 & -0.28 & -0.99 & & 107 & -0.01 & -0.68 & 0.78 & 5509 & 0.003 & 3195 & 0.009 & 3650 & 0.187 & 6453 & 0.215 \\
\hline Sagittal head & 107 & 0.15 & -0.49 & 0.7 & 133 & -0.09 & -0.80 & 0.45 & 77 & 0.06 & -0.62 & 0. & 107 & -0.05 & -0.76 & 0.57 & 5769 & 0.012 & 3783 & 0.345 & 3792 & 0.358 & 6873 & 0.649 \\
\hline Mandibular arc & 106 & 0.10 & -0.24 & 0.53 & 133 & 0.13 & -0.57 & 0.75 & 75 & -0.22 & -0.54 & 0. & 106 & -0.17 & -0.73 & 0.43 & 6838 & 0.691 & 3756 & 0.527 & 2843 & 0.001 & 5923 & 0.034 \\
\hline Lip-chi & 106 & 0.12 & -0.83 & 0.2 & 1 & 0. & -0.24 & & & -0.37 & -1.06 & & 107 & -0.12 & -0.80 & & & 0.0 & 3303 & 0.02 & & 0.0 & 5390 & \\
\hline & 102 & 0.2 & -0 . & & & 0. & -0 . & & 7 & & -0 & & 1 & -0 & -0 & & & 0.8 & & & & & & \\
\hline & 10 & -0 . & -0 & & 1 & 0 & & & 7 & & -0 & & 16 & -0 & -1 & & 65 & & & & & 0. & 4872 & $<0.001 *$ \\
\hline & 106 & -0.57 & -0 & -0 & 133 & 0. & -0 & & 75 & & -0 & 0.55 & 107 & 0. & -0.42 & 0.73 & 3080 & $<0.001 *$ & 2999 & 0.004 & 3238 & 0.034 & 6762 & 0.508 \\
\hline Thigh circumference & 106 & -0.61 & -1.29 & -0.11 & 133 & 0.20 & -0.25 & 0.79 & 77 & -0.37 & -0.82 & 0.35 & 106 & 0.25 & -0.26 & 0.79 & 3061 & $<0.001 *$ & 2897 & 0.001 & & & 6982 & 0.900 \\
\hline Calf circumference & 106 & -0.34 & -0.83 & 0.22 & 132 & 0.22 & -0.23 & 0.74 & 77 & -0.29 & -0.88 & & 107 & 0.06 & -0.54 & & 4173 & $<0.001 *$ & 3527 & 0.09 & 3910 & 0.628 & 5678 & 0.009 \\
\hline Ankle circum & 106 & 0.00 & -0.35 & 0. & 13 & 0 & -0.25 & & 77 & -0.2 & -0.58 & & 10 & -0.19 & -0.45 & & 6167 & 0.1 & 3957 & 0. & 3229 & 0.016 & 5040 & $<0.001$ \\
\hline & 99 & $-0 . c$ & -0.55 & 0.7 & 105 & 0.23 & -0.35 & 1. & 74 & -0 & -0.97 & 0 . & 100 & 0.04 & -0.65 & 0.50 & 4547 & 0.1 & 2866 & & 2628 & 0.001 & 4270 & \\
\hline Foot breadth & 100 & -0.07 & -0.69 & 0.68 & 107 & 0.32 & -0.15 & 1.02 & 75 & -0.19 & -0.81 & 0.51 & 101 & -0.14 & -0.77 & 0.36 & 4056 & 0.003 & 3755 & 0.923 & 3345 & 0.222 & 3389 & $<0.001 *$ \\
\hline
\end{tabular}

Medians (M), interquartile ranges (IQR), absolute body weight in $\mathrm{kg}$, absolute length dimensions in $\mathrm{cm}, P<0.05$ after adjustment using the false discovery rate is colored and indicates the following direction: ADP $<$ HCS, ADP $>$ HCS, males $>$ females, males $<$ females. ${ }^{*} P<0.05$ after Bonferroni correction. 
Table 3. Differences in body measurements between alcohol-dependent in-patients (ADP) without and those with prospective 12-month alcohol-related readmission.

\begin{tabular}{|c|c|c|c|c|c|c|c|c|c|c|c|c|c|c|c|c|c|c|c|c|}
\hline & \multicolumn{10}{|c|}{ 12-Month Readmission in Male ADP } & \multicolumn{10}{|c|}{ 12-Month Readmission in Female ADP } \\
\hline & \multicolumn{4}{|c|}{ No } & \multicolumn{4}{|c|}{ Yes } & \multirow[b]{2}{*}{$u$} & & \multicolumn{4}{|c|}{ No } & \multicolumn{4}{|c|}{ Yes } & \multirow[b]{2}{*}{$u$} & \multirow[b]{2}{*}{$P$} \\
\hline & $N$ & $\mathbf{M}$ & IC & & $N$ & M & & & & $P$ & $N$ & $\mathbf{M}$ & IC & & $N$ & $\mathrm{M}$ & & & & \\
\hline \multicolumn{21}{|l|}{ Absolute measure } \\
\hline Body height & 46 & 177.7 & 173.0 & 181.0 & 61 & 176.2 & 171.0 & 181.4 & 1200 & 0.201 & 45 & 164.3 & 160.5 & 169.0 & 32 & 164.8 & 163.0 & 169.2 & 628 & 0.339 \\
\hline Body weight & 45 & 74.2 & 68.1 & 83.3 & 61 & 80.2 & 71.8 & 90.4 & 1098 & 0.079 & 47 & 68.2 & 57.9 & 76.7 & 30 & 65.7 & 60.5 & 81.3 & 650 & 0.562 \\
\hline Head circumference & 46 & 57.3 & 56.2 & 58.0 & 61 & 57.5 & 56.4 & 59.0 & 1209 & 0.221 & 47 & 54.5 & 54.0 & 56.0 & 32 & 55.5 & 53.7 & 57.0 & 593 & 0.111 \\
\hline Bitragion head arc & 46 & 37.0 & 36.0 & 37.7 & 61 & 36.3 & 35.4 & 37.5 & 1140 & 0.097 & 47 & 35.5 & 34.5 & 36.2 & 32 & 35.5 & 35.0 & 36.5 & 640 & 0.260 \\
\hline Sagittal head arc & 46 & 36.0 & 35.0 & 37.4 & 61 & 36.0 & 34.7 & 37.7 & 1381 & 0.890 & 47 & 34.8 & 33.0 & 36.0 & 32 & 35.2 & 34.1 & 36.3 & 608 & 0.149 \\
\hline Mandibular arc & 46 & 24.2 & 23.4 & 25.5 & 60 & 23.6 & 22.8 & 25.0 & 1120 & 0.096 & 47 & 22.0 & 21.0 & 23.0 & 30 & 22.3 & 22.0 & 23.1 & 582 & 0.194 \\
\hline Lip-chin distance & 46 & 5.5 & 4.9 & 6.0 & 60 & 5.5 & 5.0 & 5.8 & 1306 & 0.635 & 47 & 5.0 & 4.2 & 5.5 & 32 & 5.0 & 4.6 & 5.5 & 710 & 0.668 \\
\hline Wrist circumference & 44 & 17.7 & 16.5 & 18.2 & 58 & 17.7 & 16.9 & 18.5 & 1131 & 0.327 & 46 & 15.9 & 15.5 & 16.8 & 29 & 16.1 & 15.5 & & 590 & 0.401 \\
\hline Waist circumference & 46 & 92.0 & 86.4 & 97.7 & 60 & 95.6 & 88.3 & 105.5 & 1115 & 0.091 & 45 & 87.0 & 81.0 & 93.5 & 32 & 86.8 & 77.0 & 101.0 & 713 & 0.938 \\
\hline Hip circumference & 46 & 92.6 & 88.0 & 99.4 & 60 & 95.3 & 90.0 & 100.8 & 1201 & 0.252 & 45 & 96.5 & 91.7 & 103.0 & 32 & 95.2 & 89.8 & 108.1 & 717 & 0.971 \\
\hline Thigh circumference & 46 & 52.9 & 49.8 & 56.5 & 60 & 56.6 & 53.5 & 60.2 & 911 & 0.003 & 47 & 56.0 & 53.0 & 62.5 & 32 & 56.9 & 54.0 & 61.0 & 709 & 0.668 \\
\hline Calf ci & 46 & 36.2 & 34.5 & 37. & 60 & 37.4 & & 39.7 & 998 & & 47 & 35. & 33.3 & & 32 & & 34.2 & & 686 & 0.506 \\
\hline Ankle circumference & 46 & 26.0 & 25.0 & 27.0 & 60 & 26.5 & 25.5 & 27.5 & 1109 & 0.084 & 47 & 24.1 & 23.0 & 26.0 & 32 & 24.5 & 23.8 & 25.3 & 672 & 0.423 \\
\hline Foot length & 41 & 26.0 & 25.0 & 27.2 & 58 & 26.1 & 25.3 & 26.8 & 1183 & 0.966 & 45 & 23.7 & 22.7 & 24.7 & 32 & 24.1 & 23.5 & 24.8 & 604 & 0.229 \\
\hline Foot breadth & 42 & 9.7 & 9.5 & 10.0 & 58 & 9.7 & 9.4 & 10.2 & 1180 & 0.788 & 46 & 9.1 & 8.8 & 9.5 & 32 & 9.1 & 8.9 & & 731 & 0.959 \\
\hline \multicolumn{21}{|c|}{ Measure divided by body height } \\
\hline Head circumference & 46 & 0.32 & 0.31 & 0.33 & 61 & 0.33 & 0.32 & 0.34 & 1071 & 0.036 & 45 & 0.33 & 0.32 & 0.34 & 32 & 0.33 & 0.32 & 0.35 & 705 & 0.877 \\
\hline & 46 & 0.21 & 0.20 & 0.21 & 61 & 0.21 & 0.20 & 0.22 & 1304 & 0.531 & 45 & 0.22 & 0.21 & 0.22 & 32 & 0.22 & 0.21 & 0.22 & 705 & 0.873 \\
\hline & 46 & 0.20 & 0.20 & 0.2 & 61 & 0.21 & & & 1285 & & 45 & 0.21 & & & 32 & & 0.20 & & 694 & 0.784 \\
\hline Mandibu & 46 & 0.14 & 0.13 & 0.15 & 60 & 0.14 & 0.13 & 0.14 & 1255 & 0.426 & 45 & 0.13 & 0.13 & 0.14 & 30 & 0.13 & 0.13 & 0.14 & 628 & 0.611 \\
\hline Lip-chin & 46 & 0.03 & 0.03 & 0.03 & 60 & 0.03 & 0.03 & 0.03 & 1357 & 0.883 & 45 & 0.03 & 0.03 & & 32 & 0.03 & 0.03 & 0.03 & 689 & 0.749 \\
\hline & 44 & 0.10 & 0.09 & 0.10 & 58 & 0.10 & 0.10 & 0.10 & 1034 & 0.10 & 44 & 0.10 & 0.09 & & 29 & 0.10 & 0.09 & 0.10 & 620 & 0.839 \\
\hline & 46 & 0 & 0.49 & 0.5 & 60 & & & & 1026 & & 4 & 0.5 & 49 & & 32 & & 0.46 & 0.59 & 63 & 0.785 \\
\hline Hip cir & 46 & 0.52 & 0.49 & 0.56 & 60 & 0.54 & 0.52 & 0.57 & 1076 & 0.052 & 43 & 0.60 & 0.54 & 0.64 & 32 & 0.57 & 0.54 & 0.64 & 660 & 0.764 \\
\hline & 46 & 0.30 & 0.28 & 0.32 & 60 & 0.32 & 0.30 & 0. & 811 & $<0.001$ & 45 & 0.34 & 0.33 & & 32 & & 0.33 & & 690 & 0.753 \\
\hline & 46 & 0.21 & 0.19 & 0.22 & 60 & 0.21 & 0.20 & 0.23 & 939 & 0.005 & 45 & 0.22 & 0.20 & & 32 & 0.22 & 0.21 & 0. & 677 & 0.653 \\
\hline Ankle cir & 46 & 0. & 0.1 & 0. & 60 & & & & 1017 & & 4 & 0.1 & & & 32 & & 0.14 & & 714 & 0.946 \\
\hline & 41 & 0.15 & 0.14 & 0.15 & 58 & 0.1 & 0.14 & 0. & 1050 & 0.3 & 42 & 0.14 & 0.14 & & 32 & 0.15 & 0.14 & 0.15 & 612 & 0.513 \\
\hline Foot bread & 42 & 0.05 & 0.05 & 0.06 & 58 & 0.06 & 0.05 & 0.06 & 1092 & 0.37 & 43 & 0.06 & 0.05 & 0.0 & 32 & 0.06 & 0.05 & 0.06 & 603 & 0.363 \\
\hline \multicolumn{21}{|c|}{ Measure residualized on body height } \\
\hline Head circumference & 46 & -0.14 & -0.50 & 0.4 & 61 & 0.2 & -0.38 & 0.76 & 1104 & 0.05 & 45 & -0.58 & -1.02 & 0.07 & 32 & -0.34 & -0.90 & 0.39 & 609 & 0.251 \\
\hline & 46 & 0.01 & -0.45 & 0.31 & 61 & -0.26 & -0.99 & 0.29 & 1173 & & 45 & -0.49 & -0.99 & & 32 & -0.23 & -0.95 & 0.27 & 677 & 0.653 \\
\hline & 46 & 0.15 & -0.44 & 0.78 & 61 & 0.16 & -0.54 & 0.77 & 1351 & 0. & 45 & -0.02 & -0.62 & 0 & 32 & 0.08 & -0.58 & 0.69 & 637 & 0.391 \\
\hline & 46 & & -0.21 & 0.7 & 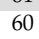 & & -0.35 & & & & & -0.33 & -0.69 & & 30 & -0.18 & -0.46 & & 年 & 0.482 \\
\hline & 4 & & -0 & 1. & 60 & & -0 & & & & 45 & & -1.23 & & 32 & -0.39 & -0 & & 681 & 0.687 \\
\hline Wrist c & 44 & & -0.18 & 0.52 & 58 & & -0.03 & & 1040 & & 44 & -0.04 & -0.16 & & 29 & 0.01 & -0.40 & 0.22 & 628 & 0.910 \\
\hline Waist c & 46 & -0.19 & -0.49 & 0.34 & 60 & & -0.31 & 0.89 & 1045 & 0. & 43 & -0.05 & -0.60 & 0. & 32 & $\begin{array}{l}-0.30 \\
-0.30\end{array}$ & -0.97 & 0.69 & 664 & 0.793 \\
\hline & 46 & -0.68 & -1.11 & -0. & 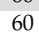 & & -0.92 & & 1189 & & 43 & -0.28 & -0.90 & & 32 & -0.38 & -0.87 & & 682 & \\
\hline & 46 & & -1 & -0 . & 60 & & & & 8 & & 45 & & -0 & & 32 & & -0.73 & 0.34 & 677 & 0.653 \\
\hline & 46 & -0.61 & -1.21 & 0.01 & 60 & -0.22 & -0.60 & 0.40 & 964 & 0.0 & 45 & -0.42 & -0.92 & 0.43 & 32 & -0.09 & -0.87 & 0.44 & 663 & 0.552 \\
\hline Ankle circum & 46 & -0.10 & $\begin{array}{l}-0.39 \\
-0.39\end{array}$ & 0.28 & 60 & 0.15 & -0.26 & 0.68 & 1041 & & 45 & -0.22 & -0.64 & 0.20 & 32 & -0.21 & -0.53 & 0.13 & 704 & 0.865 \\
\hline & 41 & -0.03 & -0.79 & 0.51 & 58 & 0.09 & -0.48 & & 1054 & & 42 & -0.44 & -0.93 & & 32 & -0.07 & -1.19 & 0.21 & 622 & 058 \\
\hline Foot breadth & 42 & -0.40 & -0.69 & 0.72 & 58 & 0.10 & -0.73 & 0.66 & 1108 & 0.442 & 43 & -0.19 & -0.70 & 0.54 & 32 & -0.31 & -0.94 & 0.27 & 623 & 0.486 \\
\hline
\end{tabular}

Medians (M), interquartile ranges (IQR), absolute body weight in $\mathrm{kg}$, absolute length dimensions in $\mathrm{cm}, P<0.05$ after adjustment using the false discovery rate is colored and indicates the following direction: ADP without 12-month readmission $<$ ADP with 12-month readmission (no $P<0.05$ after Bonferroni correction). 
Table 4. Differences in body measurements between alcohol-dependent in-patients (ADP) without and those with prospective 24-month alcohol-related readmission.

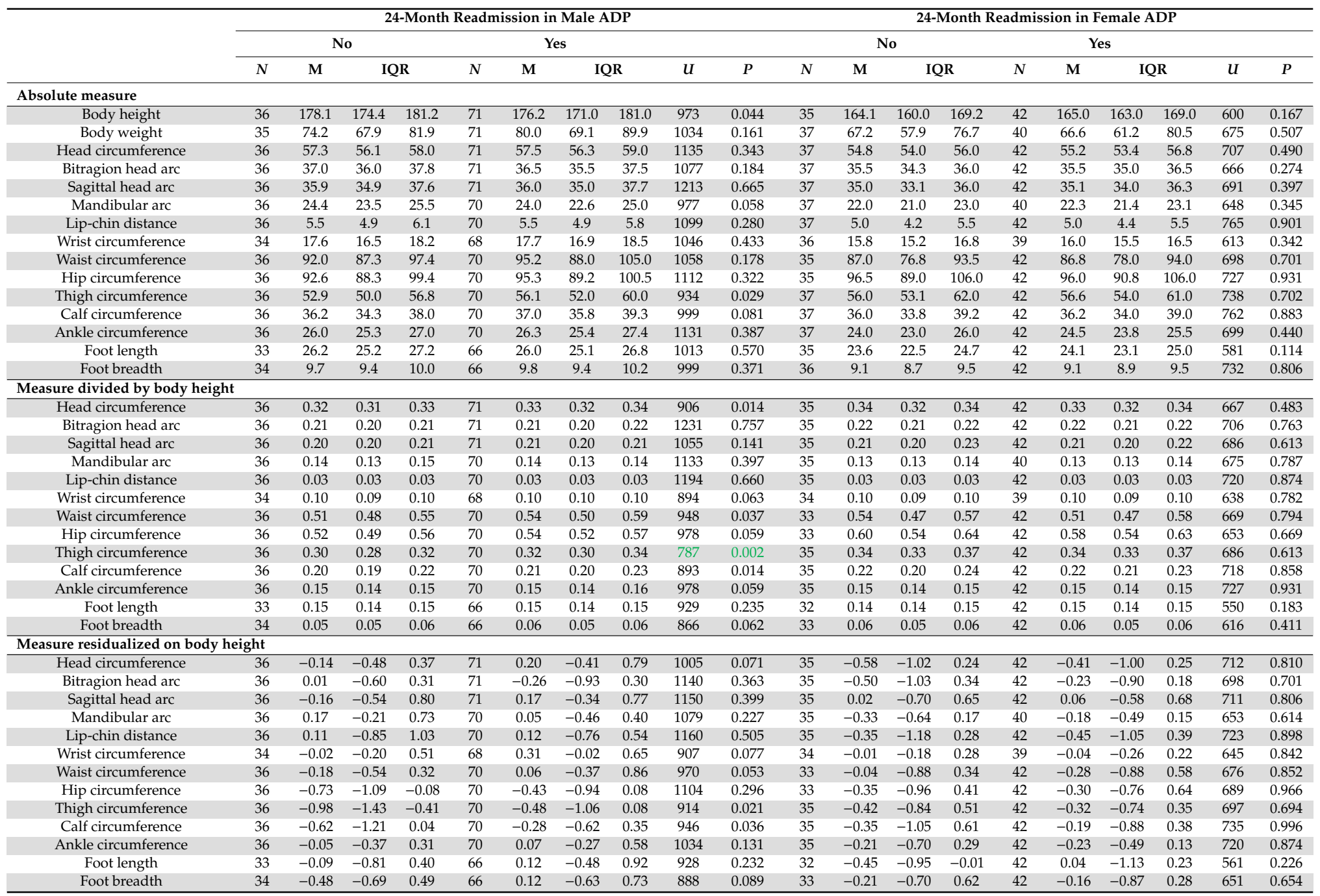

Medians $(\mathrm{M})$, interquartile ranges $(\mathrm{IQR})$, absolute body weight in $\mathrm{kg}$, absolute length dimensions in $\mathrm{cm}, P<0.05$ after adjustment using the false discovery rate is colored and indicates the following direction: ADP without 24-month readmission $<$ ADP with 24-month readmission (no $P<0.05$ after Bonferroni correction). 
Table 5. Spearman correlations of absolute body measurements with days to first and number of alcohol-related readmissions in alcohol-dependent in-patients (ADP).

\begin{tabular}{|c|c|c|c|c|c|c|c|c|c|c|c|c|c|c|c|c|c|c|}
\hline & \multirow[b]{3}{*}{$N$} & \multicolumn{4}{|c|}{ Male ADP 12-Month Readmission } & \multicolumn{4}{|c|}{ Male ADP 24-Month Readmission } & \multirow[b]{3}{*}{$N$} & \multicolumn{4}{|c|}{ Female ADP 12-Month Readmission } & \multicolumn{4}{|c|}{ Female ADP 24-Month Readmission } \\
\hline & & \multicolumn{2}{|c|}{ Latency (Days) } & \multicolumn{2}{|c|}{ Number } & \multicolumn{2}{|c|}{ Latency (Days) } & \multicolumn{2}{|c|}{ Number } & & \multicolumn{2}{|c|}{ Latency (Days) } & \multicolumn{2}{|c|}{ Number } & \multicolumn{2}{|c|}{ Latency (Days) } & \multicolumn{2}{|c|}{ Number } \\
\hline & & $\rho$ & $P$ & $\rho$ & $P$ & $\rho$ & $P$ & $\rho$ & $P$ & & $\rho$ & $P$ & $\rho$ & $P$ & $\rho$ & $P$ & $\rho$ & $P$ \\
\hline \multicolumn{19}{|l|}{ Absolute measure } \\
\hline Body height & 107 & 0.091 & 0.349 & -0.082 & 0.400 & 0.122 & 0.210 & -0.131 & 0.179 & 77 & -0.041 & 0.722 & 0.123 & 0.286 & -0.098 & 0.399 & 0.152 & 0.187 \\
\hline Body weight & 106 & -0.150 & 0.126 & 0.175 & 0.072 & -0.140 & 0.152 & 0.188 & 0.054 & 77 & -0.028 & 0.807 & 0.102 & 0.379 & -0.062 & 0.592 & 0.104 & 0.368 \\
\hline Head circumference & 107 & -0.131 & 0.178 & 0.140 & 0.151 & -0.126 & 0.196 & 0.160 & 0.099 & 79 & -0.132 & 0.245 & 0.167 & 0.141 & -0.090 & 0.433 & 0.149 & 0.189 \\
\hline Bitragion head arc & 107 & 0.195 & 0.044 & -0.179 & 0.065 & 0.190 & 0.050 & -0.147 & 0.131 & 79 & -0.058 & 0.614 & 0.073 & 0.525 & -0.037 & 0.743 & 0.099 & 0.385 \\
\hline Sagittal head arc & 107 & -0.006 & 0.952 & 0.120 & 0.217 & -0.019 & 0.848 & 0.162 & 0.095 & 79 & -0.178 & 0.116 & 0.206 & 0.069 & -0.169 & 0.137 & 0.192 & 0.090 \\
\hline Mandibular arc & 106 & 0.122 & 0.212 & -0.141 & 0.149 & 0.141 & 0.151 & -0.142 & 0.147 & 77 & -0.078 & 0.503 & 0.115 & 0.319 & -0.058 & 0.618 & 0.102 & 0.376 \\
\hline Lip-chin distance & 106 & 0.040 & 0.686 & -0.094 & 0.339 & 0.060 & 0.542 & -0.133 & 0.174 & 79 & -0.040 & 0.724 & 0.002 & 0.986 & 0.008 & 0.945 & -0.020 & 0.865 \\
\hline Wrist circumference & 102 & -0.121 & 0.225 & 0.110 & 0.270 & -0.116 & 0.244 & 0.120 & 0.230 & 75 & -0.049 & 0.679 & 0.103 & 0.378 & -0.096 & 0.411 & 0.111 & 0.342 \\
\hline Waist circumference & 106 & -0.106 & 0.279 & 0.142 & 0.147 & -0.096 & 0.326 & 0.146 & 0.135 & 77 & 0.043 & 0.708 & 0.011 & 0.928 & 0.010 & 0.930 & 0.044 & 0.706 \\
\hline Hip circumference & 106 & -0.095 & 0.334 & 0.127 & 0.196 & -0.091 & 0.351 & 0.151 & 0.123 & 77 & 0.050 & 0.666 & -0.012 & 0.920 & 0.035 & 0.761 & -0.029 & 0.801 \\
\hline Thigh circumference & 106 & -0.260 & 0.007 & 0.274 & 0.004 & -0.240 & 0.013 & 0.257 & 0.008 & 79 & -0.055 & 0.628 & 0.118 & 0.301 & -0.080 & 0.484 & 0.095 & 0.405 \\
\hline Calf circumference & 106 & -0.270 & 0.005 & 0.199 & 0.041 & -0.249 & 0.010 & 0.181 & 0.063 & 79 & -0.050 & 0.660 & 0.124 & 0.276 & -0.050 & 0.664 & 0.060 & 0.598 \\
\hline Ankle circumference & 106 & -0.123 & 0.210 & 0.140 & 0.153 & -0.097 & 0.322 & 0.087 & 0.376 & 79 & -0.062 & 0.588 & 0.118 & 0.302 & -0.090 & 0.431 & 0.094 & 0.408 \\
\hline Foot length & 99 & -0.030 & 0.765 & 0.006 & 0.953 & -0.009 & 0.929 & -0.026 & 0.797 & 77 & -0.088 & 0.449 & 0.185 & 0.108 & -0.150 & 0.193 & 0.215 & 0.061 \\
\hline Foot breadth & 100 & -0.110 & 0.276 & 0.039 & 0.700 & -0.131 & 0.195 & 0.092 & 0.363 & 78 & 0.062 & 0.588 & 0.002 & 0.987 & 0.015 & 0.899 & -0.004 & 0.974 \\
\hline \multicolumn{19}{|c|}{ Measure divided by body height } \\
\hline Head circumference & 107 & -0.184 & 0.058 & 0.200 & 0.038 & -0.203 & 0.036 & 0.260 & 0.007 & 77 & -0.044 & 0.702 & -0.016 & 0.891 & 0.024 & 0.837 & -0.048 & 0.676 \\
\hline Bitragion head arc & 107 & 0.108 & 0.267 & -0.101 & 0.300 & 0.079 & 0.416 & -0.040 & 0.686 & 77 & 0.013 & 0.911 & -0.060 & 0.605 & 0.058 & 0.614 & -0.051 & 0.660 \\
\hline Sagittal head arc & 107 & -0.040 & 0.684 & 0.145 & 0.136 & -0.069 & 0.478 & 0.224 & 0.020 & 77 & -0.100 & 0.386 & 0.060 & 0.605 & -0.054 & 0.642 & 0.026 & 0.824 \\
\hline Mandibular arc & 106 & 0.041 & 0.674 & -0.079 & 0.423 & 0.050 & 0.612 & -0.064 & 0.517 & 75 & -0.031 & 0.792 & 0.025 & 0.833 & 0.000 & 0.997 & 0.010 & 0.931 \\
\hline Lip-chin distance & 106 & 0.022 & 0.826 & -0.063 & 0.520 & 0.030 & 0.764 & -0.083 & 0.397 & 77 & -0.047 & 0.687 & 0.000 & 0.999 & 0.002 & 0.985 & -0.019 & 0.869 \\
\hline Wrist circumference & 102 & -0.175 & 0.079 & 0.159 & 0.109 & -0.187 & 0.060 & 0.187 & 0.060 & 73 & 0.034 & 0.776 & 0.010 & 0.9 & 0.010 & 0.934 & -0.004 & 0.973 \\
\hline Waist circumference & 106 & -0.151 & 0.123 & 0.183 & 0.060 & -0.149 & 0.128 & 0.192 & 0.049 & 75 & 0.065 & 0.577 & -0.033 & 0.777 & 0.064 & 0.584 & -0.022 & 0.852 \\
\hline Hip circumference & 106 & -0.161 & 0.100 & 0.180 & 0.064 & -0.164 & 0.093 & 0.211 & 0.030 & 75 & 0.054 & 0.644 & -0.037 & 0.750 & 0.052 & 0.659 & -0.062 & 0.598 \\
\hline Thigh circumference & 106 & -0.308 & 0.001 & 0.315 & 0.001 & -0.303 & 0.002 & 0.320 & 0.001 & 77 & 0.009 & 0.935 & 0.040 & 0.729 & -0.001 & 0.994 & 0.003 & 0.979 \\
\hline Calf circumference & 106 & -0.284 & 0.003 & 0.212 & 0.029 & -0.277 & 0.004 & 0.223 & 0.02 & 77 & -0.047 & 0.685 & 0.098 & 0.395 & -0.034 & 0.769 & 0.023 & 0.845 \\
\hline Ankle circumference & 106 & -0.181 & 0.063 & 0.178 & 0.068 & -0.172 & 0.079 & 0.153 & 0.117 & 77 & -0.017 & 0.884 & 0.038 & 0.740 & -0.028 & 0.809 & 0.002 & 0.984 \\
\hline Foot length & 99 & -0.122 & 0.230 & 0.090 & 0.374 & -0.129 & 0.201 & 0.103 & 0.312 & 74 & -0.086 & 0.464 & 0.129 & 0.272 & -0.159 & 0.175 & 0.177 & 0.131 \\
\hline Foot breadth & 100 & -0.142 & 0.159 & 0.084 & 0.407 & -0.178 & 0.076 & 0.163 & 0.105 & 75 & 0.115 & 0.325 & -0.098 & 0.402 & 0.100 & 0.391 & -0.121 & 0.300 \\
\hline Measure residualized on & height & & & & & & & & & & & & & & & & & \\
\hline Head circumference & 107 & -0.184 & 0.058 & 0.196 & 0.043 & -0.187 & 0.054 & 0.232 & 0.016 & 77 & -0.115 & 0.320 & 0.112 & 0.331 & -0.058 & 0.614 & 0.089 & 0.439 \\
\hline Bitragion head arc & 107 & 0.183 & 0.059 & -0.171 & 0.078 & 0.170 & 0.080 & -0.120 & 0.217 & 77 & -0.016 & 0.891 & 0.002 & 0.986 & 0.019 & 0.871 & 0.020 & 0.864 \\
\hline Sagittal head arc & 107 & -0.021 & 0.832 & 0.129 & 0.184 & -0.042 & 0.669 & 0.187 & 0.054 & 77 & -0.134 & 0.246 & 0.136 & 0.237 & -0.114 & 0.324 & 0.123 & 0.287 \\
\hline Mandibular arc & 106 & 0.068 & 0.487 & -0.099 & 0.311 & 0.080 & 0.412 & -0.089 & 0.366 & 75 & -0.038 & 0.746 & & 0.716 & -0.015 & 0.900 & 0.031 & 0.793 \\
\hline Lip-chin distance & 106 & 0.023 & 0.81 & -0.069 & 0.48 & 0.03 & 0.705 & -0.095 & 0.3 & 77 & -0.051 & 0.659 & 0.005 & 0.9 & -0.001 & 0.995 & -0.017 & 0.885 \\
\hline Wrist circumference & 102 & -0.171 & 0.085 & 0.158 & 0.113 & -0.181 & 0.068 & 0.183 & 0.066 & 73 & 0.023 & 0.846 & 0.021 & 0.857 & -0.001 & 0.994 & 0.006 & 0.956 \\
\hline Waist circumference & 106 & -0.144 & 0.140 & 0.178 & 0.068 & -0.141 & 0.149 & 0.187 & 0.056 & 75 & 0.069 & 0.556 & -0.026 & 0.824 & 0.058 & 0.620 & -0.012 & 0.919 \\
\hline Hip circumference & 106 & -0.101 & 0.302 & 0.132 & 0.177 & -0.097 & 0.322 & 0.155 & 0.114 & 75 & 0.036 & 0.756 & -0.001 & 0.990 & 0.020 & 0.866 & -0.017 & 0.883 \\
\hline Thigh circumference & 106 & -0.269 & 0.005 & 0.282 & 0.00 & -0.249 & 0.010 & 0.267 & 0.0 & 77 & -0.058 & 0.614 & 0.125 & 0.280 & -0.083 & 0.471 & 0.101 & 0.380 \\
\hline Calf circumference & 106 & -0.280 & 0.004 & 0.209 & 0.032 & -0.265 & 0.006 & 0.204 & 0.036 & 77 & -0.049 & 0.671 & 0.117 & 0.311 & -0.043 & 0.708 & 0.047 & 0.686 \\
\hline Ankle circumference & 106 & -0.165 & 0.092 & 0.166 & 0.089 & -0.147 & 0.132 & 0.125 & 0.202 & 77 & -0.018 & 0.875 & 0.052 & 0.652 & -0.042 & 0.717 & 0.026 & 0.822 \\
\hline Foot length & 99 & -0.118 & 0.244 & 0.088 & 0.387 & -0.127 & 0.210 & 0.102 & 0.314 & 74 & -0.076 & 0.521 & 0.117 & 0.320 & -0.147 & 0.212 & 0.166 & 0.158 \\
\hline Foot breadth & 100 & -0.139 & 0.167 & 0.080 & 0.431 & -0.173 & 0.085 & 0.156 & 0.122 & 75 & 0.099 & 0.397 & -0.067 & 0.571 & 0.068 & 0.562 & -0.078 & 0.508 \\
\hline
\end{tabular}

$P<0.05$ after adjustment using the false discovery rate is colored and indicates the following direction: higher values of body measurements correspond to a shorter latency to the first readmission and to a higher number of readmissions for the 12- and 24-month follow-up periods (no $P<0.05$ after Bonferroni correction). 
Absolute Body Measurements: Male sex was related to higher body weight and height, head circumference, lengths of bitragion and sagittal head arc and mandibular arc, lip-chin distance, wrist, waist, and ankle circumference, and foot length and breadth in both groups and to a longer calf circumference in the healthy control subjects. Body Measurements Divided by Height: Male sex was linked to lower head circumference values, lengths of bitragion and sagittal head arc, a higher wrist circumference, and lower hip and thigh circumferences in both groups, to a smaller calf circumference and a longer foot length in alcohol-dependent in-patients, and to a higher waist circumference in healthy control subjects. Body Measurements Residualized on Height: Male sex was associated with longer head and wrist circumference in both groups, with a longer mandibular arc, shorter thigh circumference, and higher foot length in alcohol-dependent in-patients, and with longer lip-chin distance, waist and ankle circumference, and foot breadth in healthy control subjects.

Many of these sex differences remained significant after the Bonferroni correction.

3.5. Basic Human Body Dimensions: Exploratory Analysis of Correlations with 2D:4D, the Age of Onset of Regular Alcohol Drinking and First In-Patient Treatment Due to Alcohol Problems, Total Lifetime Drinking and Daily Ethanol Intake, and Inter-Correlations

The analyses revealed no convincing pattern of significant relationships between 2D:4D and absolute body measurements, body measurements divided by height, or body measurements residualized on height (no significant correlation except for seven tests with $P>0.039$, Table A1 in Appendix A).

The age of onset of regular drinking (i.e., daily over at least 7 days) and the age of the subjects' first in-patient treatment due to alcohol problems correlated positively and significantly with many absolute, relative, and residualized body measurements in both male and female alcohol-dependent in-patients; however, there were also significant negative correlations with the lengths of sagittal head arc and thigh circumference in male patients (Table A2 in Appendix A).

Absolute Body Measurements: In male alcohol-dependent in-patients, the age of onset of regular drinking and/or the age of first in-patient treatment due to alcohol problems correlated positively with lengths of mandibular arc, waist, hip, and ankle circumferences and negatively with sagittal head arc and thigh circumference. Body Measurements Divided by Height: The age of onset of regular drinking and/or the age of first in-patient treatment due to alcohol problems correlated positively with lengths of mandibular arc and waist and ankle circumference and negatively with sagittal head arc and thigh circumference in male alcohol-dependent in-patients and positively with wrist and ankle circumference and foot length and breadth in female alcohol-dependent in-patients. Body Measurements Residualized on Height: The age of onset of regular drinking and/or the age of first in-patient treatment due to alcohol problems correlated positively with lengths of mandibular arc and waist, hip, and ankle circumferences and negatively with sagittal head arc and thigh circumference in male alcohol-dependent in-patients and positively with bitragion head arc, wrist and ankle circumference, and foot length and breadth in female alcohol-dependent in-patients.

Total lifetime drinking and daily ethanol intake did not significantly correlate with absolute, relative, or residualized body measurements in male and female alcohol-dependent in-patients, except for a significant negative correlation between total lifetime drinking and absolute lip-chin distance in male patients and between daily ethanol intake and absolute, relative, and residualized mandibular arc length in male and female patients $(P>0.025)$ (Table A3 in Appendix A).

We found multiple significant inter-correlations among the absolute body measurements (Tables A4 and A5 in Appendix A), the body measurements divided by height (Tables A6 and A7 in Appendix A), and the body measurements residualized on height (Tables A8 and A9 in Appendix A).

\section{Discussion}

As far as we know, this is the first systematic and comprehensive anthropomorphic study that determined the associations between basic human body dimensions and alcohol dependence. 
Separately for both sexes, we have found many shorter body measurements in alcohol-dependent in-patients than in healthy control subjects. These results are supported by two earlier studies that indicated that participants recruited from alcoholism in-patient treatment programs have lower body height, body weight, and thigh volume than controls [61,62], although it should be noted that these projects focused primarily on body composition and sex differences in alcohol consumption. Moreover, we showed here for the first time that in male alcohol-dependent in-patients, a higher thigh circumference may predict a worse 12-month outcome with a higher risk, shorter latency to the first alcohol-related readmission, and a higher number of alcohol-related readmissions.

We were able to verify many of the a priori expected sexual dimorphisms with shorter absolute and residualized on height physical dimensions in females than males. These results confirm the assumption that the body dimensions may provide insight into the organizational effects of sex hormones. Hence, the associations between sexually dimorphic body measurements and alcohol dependence observed here may support that the organizational effects of sex hormones are involved in alcohol dependence. Interestingly, the absolute, relative, and residualized body measurements did not convincingly correlate with 2D:4D in any subgroup. The independence of these factors may suggest that, rather than prenatal sex hormones, the pubertal (or later) sex hormones organize the physical dimensions analyzed in this study. Support of this idea comes from a rodent study that failed to show consistent effects of prenatal androgen receptor modulation on head size and body length [11]. It is therefore more likely that pubertal (or even later), rather than prenatal sex hormone effects, account for the differences between alcohol-dependent in-patients and healthy control subjects observed here. Moreover, the smaller body height of alcohol-dependent males in comparison to healthy control males detected here is contradictory to the expectations based on findings from the TTT. In males, a male co-twin in comparison to a female co-twin reduces both the risk for later alcohol dependence [36] and body height [63], although the effect sizes were small in both investigations. However, this entails the prediction of larger body height in alcohol-dependent males. The opposite finding in this study further corroborates that, rather than prenatal sex hormones, the pubertal (or later) sex hormones organize the physical dimensions analyzed here. It may also indicate that other factors as discussed in the following section are involved.

\subsection{Possible Reasons for Smaller Body Dimensions in Alcohol Dependence}

We detected many shorter absolute, relative, and residualized body measurements in alcohol-dependent in-patients than in healthy control subjects. Shorter measurements for most of the absolute and residualized parameters were also present in females than in males. At first glance, the shorter body measurements in the alcohol dependence group (compared to the control group), in light of the shorter values in females than in males, might appear contradictory to the higher prevalence of alcohol dependence in males than in females [1]. However, different reasons might account for these results.

Prepubertal boys show lower estradiol levels than prepubertal girls [64], which entails the later onset of puberty in males. Sex differences in bone mass, size, and strength strongly increase during puberty, with higher values in males compared to females [65,66]. Males are taller and develop longer bones than females because their growth spurts last longer before the epiphyses fuse, which results from the later rise and lower peak levels of estradiol (see [67], regarding sex differences in estrogen and epiphyseal fusing). Thus, lifelong smaller body measurements, which were observed here in alcohol dependence, may result from both a later pubertal onset and factors that reinforce estrogen signaling during puberty that may prematurely terminate the growth of the body. With regard to pubertal onset, we have already shown that male alcohol-dependent in-patients and male binge drinkers report a later age at puberty onset than control males [24,29], which may result in shorter body measurements. In addition, we hypothesize a premature termination of pubertal growth in the group of alcohol-dependent in-patients. Here, we found an overlap between the age of onset of regular alcohol drinking and puberty (the age of onset of regular alcohol drinking in years: minimum to $25 \%$ percentile, 
males $14-19$, females $14-23$ ) and $17.4 \%$ of males (16 out of 92 ) and $4.5 \%$ of females ( 3 out of 67 ) stated that they had started drinking alcohol regularly before turning 18 years old. Phytoestrogens are found in many alcoholic beverages. They are produced from plants that contain estrogen-like substances [68]. In addition, low dose alcohol consumption has been shown to increase testosterone levels in both sexes $[69,70]$ and testosterone is converted to estradiol by aromatase. We speculate that the early onset of regular alcohol drinking in the groups studied here, in male, and to a lesser extent, female alcohol-dependent in-patients, may have led to a premature epiphyseal fusing via phytoestrogens and testosterone to estradiol conversion (see [67], regarding sex differences in estrogen and epiphyseal fusing, [71]). We provide some support for this model in that the age of onset of regular drinking (i.e., daily over at least 7 days) and the age of first in-patient treatment due to alcohol problems were found to correlate positively with many absolute, relative, and residualized body measurements in both male and female alcohol-dependent in-patients. However, we acknowledge that the age of onset of regular drinking was assessed retrospectively and that the correlations do not allow for causal conclusions. In a nutshell, the smaller body dimensions in alcohol dependence observed here might be explained by a shorter pubertal growth period with later ages of pubertal onset and a premature end of bone growth. Additionally, the data reported here indicate that earlier ages at the onset of regular alcohol drinking (i.e., daily over at least 7 days) in males (more than in females) may account for the stronger group differences in body measurements between males and females that were observed in this study.

Moreover, additive genetic effects explain $40 \%$ of pubertal timing in boys and $46 \%$ in girls [72], and genes involved in sex hormone signaling have previously been associated with alcohol dependence and craving for alcohol [4-6,8-10]. Hence, shared genetic variance of pubertal timing and addictive behaviors might explain why absolute, relative, and residualized body dimensions are smaller in alcohol-dependent in-patients in the analyzed cohort.

Fetal alcohol spectrum disorder (FASD) provides another explanation for the many lower values of absolute, relative, and residualized body measurements in alcohol-dependent in-patients compared to the healthy control subjects in this study. A meta-analysis reported a high prevalence of pooled alcohol or drug dependence in $54.5 \%$ of the subjects diagnosed with FASD [73]. Growth restriction including height and head circumference is a typical phenomenon in subjects with FASD, which is also present in adulthood [74]. Thus, a higher percentage of prenatal alcohol exposure in the in-patients' cohort might also account for the smaller absolute, relative, and residualized body dimensions. We have not investigated FASD in this project and future research is necessary to further test this hypothesis. However, a recent register-based study estimated a rate of FASD of 177 children per 10,000 live births [75]. Although this study might underestimate the prevalence of FASD, it is unlikely that FASD explains the observed group differences completely.

Unhealthy nutrition and poor physical activity are frequent consequences of alcohol dependence and influence body composition, particularly the muscle and fat tissues. The smaller body dimensions, including body weight and hip, thigh, and calf circumferences in the in-patients' groups, may represent direct sequelae of alcohol dependence. Surprisingly, total lifetime drinking and daily ethanol intake in the cohort of male and female in-patients did not convincingly correlate with absolute, relative, or residualized body measurements (except for some correlations with lip-chin distance and mandibular arc). This suggests that the individual alcohol intake over time does not largely account for the group differences in body measurements and their associations with prospective outcomes. However, the lack of a clear correlation between lifetime drinking history and body measurements might be specific to the investigated cohort, because the patients were exclusively recruited in psychiatric hospitals and we did not include in-patients with severe somatic disorders. Furthermore, alcohol-dependent in-patients in this study are more likely than healthy control subjects to identify as active (males: $78 \%$ versus $22 \%$, females: $77 \%$ versus $19 \%$ ) or ever-smoker (males: $92 \%$ versus $59 \%$, females: $85 \%$ versus $48 \%$ ). Both lifetime and current smoking might influence body dimensions and contribute to the effects observed here. 
In summary, the smaller body dimensions in alcohol dependence may be due to a later age of pubertal onset, a premature end of bone growth due to the effects of alcohol, such as phytoestrogens and impaired sex hormone signaling, an increased rate of FASD, and unhealthy life-style factors that include nutrition, physical activity, and smoking behavior, which are related to alcohol dependence.

\subsection{Thigh Circumference: Alcohol Dependence and Prospective Outcome}

In this study, we found shorter thigh circumference in alcohol-dependent in-patients than in healthy control subjects. Moreover, longer thigh circumference predicted a worse prospective outcome in male alcohol-dependent patients. The latter associations with outcome did not remain significant after Bonferroni correction; although they did remain significant after FDR correction, and are thus considered robust, these results should be interpreted with caution.

In the same cohort that was analyzed here, we previously showed a lower body mass index in alcohol-dependent in-patients than in healthy control subjects [29] and an association between higher body mass index and a worse outcome in the patients' group [52]. This parallels the pattern demonstrated here for thigh circumference. Thigh circumference mirrors a combination of both bone and soft tissue, which is a similarity to the body mass index. The smaller thigh circumference (in line with the lower body mass index) in alcohol-dependent in-patients compared to the healthy control subjects might be a consequence of malnutrition and/or inactivation. Because of a strong positive correlation between body mass index and thigh circumference (males: alcohol-dependent in-patients, $n=105, \rho=0.833, P<0.001$, healthy control subjects, $n=133, \rho=0.666, P<0.001$; females: alcohol-dependent in-patients, $n=75, \rho=0.730, P<0.001$, healthy control subjects, $n=105, \rho=0.807$, $P<0.001$ ), the relationship between higher thigh circumference and alcohol-related readmissions in male alcohol-dependent in-patients observed here might be explained by potential mediators of the association between higher body mass index and alcohol relapse, which we elaborated on in [52], such as $N$-acetylaspartate, appetite-regulating peptides, neuroticism, impulsivity, and temporal discounting. Both increased relapse risk and higher body mass index have been associated with low thalamic $N$-acetylaspartate [76,77] and neuroticism [78-80]. Moreover, ghrelin, which regulates energy homeostasis, influences cerebral reward pathways. Pharmacological strategies targeting ghrelin pathways are being tested in the treatment of alcohol-dependent patients [81]. Higher body mass index and obesity are associated with higher impulsivity and higher temporal discounting $[82,83]$. Thus, higher thigh circumference (indicating higher body mass index) may be related to higher impulsivity and temporal discounting with more difficulties to resist the urge to drink alcohol in risky situations. Future longitudinal studies are needed to investigate these hypotheses.

\subsection{Limitations and Strengths}

The present research project is subject to several limitations. We did not differentiate between bone and soft tissue [for a discussion of this topic in a pubertally organized marker, see [84]. This is especially critical for thigh circumference, which is clinically the most promising body dimension identified here. Future research, which might involve medical imaging techniques in addition to direct surface measurement, is needed to disentangle the different roles of bone and soft tissue. We wish to note that this limitation is true for a large part of marker research in general. Even the correlation between surface and x-ray-measurements of $2 \mathrm{D}: 4 \mathrm{D}$ as an often used biomarker is comparatively low $(r=0.45$ [85]). The related imprecision might result in an underestimation of the relationship between markers and external criteria. Moreover, we cannot conclusively determine to what extent prenatal, pubertal, and even later factors account for the observations. Here, we provided evidence in the form of associations that does not allow for drawing causal conclusions. Further studies using longitudinal designs and investigating neurobiological aspects are needed to relate the potential mechanisms underlying the development of the here quantified absolute, relative, and residualized body measurements to alcohol dependence. The field's early stage of development resulted in many statistical tests involving absolute values, ratios, and residuals. Aiming to identify 
promising markers, we decided to use the less conservative FDR method to consider the type 1 error risk. Unsurprisingly, some of the significant effects disappeared after the more conservative Bonferroni adjustment. However, the results from this research project build a solid basis and reveal which body measurements merit future research to confirm that the results can be replicated and generalized. We acknowledge that the patients' outcome data were gathered using electronic patient records; no direct resurveys have been conducted. The parameter quantified here, i.e., alcohol-related readmission, differs from alcohol relapse.

The strengths of our study include the sex- and age-balanced cohorts and the long-term follow-up of alcohol-dependent patients. We specifically addressed alcohol-related readmissions, which are substantial problems in the long-term course of alcohol dependence. We also found consistent differences between alcohol-dependent in-patients and healthy control subjects across several body dimensions and considered the absolute values, ratios, and residuals. Finally, the physical measurements are easily accessible, which facilitates the transition of the results into daily clinical routine.

\section{Conclusions}

Here, we reported consistently lower values of a line of absolute, relative, and residualized body dimensions in alcohol-dependent in-patients than in healthy control subjects across both sexes. Differences in pubertal age, the effects of alcohol drinking during puberty, such as phytoestrogens and impaired sex hormone signaling, FASD, and life-style alterations related to alcohol dependence might account for these effects. Moreover, our results may have important clinical implications, as thigh circumference has turned out to be a potential and easily measureable predictor for alcohol-related readmissions. If the results observed here can be replicated, thigh circumference might serve as a measure to assess an in-patient's risk of prospective hospital readmissions. These findings can be utilized to establish individualized treatment strategies.

Author Contributions: Conceptualization, B.L. and J.K.; methodology, B.L. and M.G.K.; validation, C.M., C.W. and J.K.; formal analysis, B.L.; resources, J.K.; data curation, B.L.; writing-original draft preparation, B.L.; writing-review and editing, M.G.K., C.M., C.W. and J.K.; project administration, B.L.

Funding: This work was supported by intramural grants from the University Hospital of the Friedrich-Alexander University Erlangen-Nürnberg (FAU). C.M. is an associated fellow of the research training group 2162 funded by the Deutsche Forschungsgemeinschaft (DFG, German Research Foundation)-270949263/GRK2162. The funders had no role in the study design, data collection, analyses, decision to publish, or preparation of the manuscript.

Acknowledgments: We thank Andreas Ahnert, Ute Hamers, and Kristina Bayerlein for the opportunity and the support to recruit in-patients at the Klinik für Psychiatrie, Sucht, Psychotherapie und Psychosomatik of the Klinikum am Europakanal. We are grateful and appreciate the support of Birgit Braun, Juliane Behrens, Sarah Kubis, Katrin Mikolaiczik, Sarah Saigali, Marina Sibach, and Petya Tanovska for recruiting patients and control subjects. We thank Sina Therese Kirsten and Terezie Sedlinská for measuring foot length and breadth and Katrin Mikolaiczik, Marcel-René Muschler, and Petya Tanovska for measuring index and ring finger lengths. Moreover, we thank the reviewers for constructive suggestions.

Conflicts of Interest: The authors declare no conflict of interest. The funders had no role in the design of the study; in the collection, analyses, or interpretation of data; in the writing of the manuscript, or in the decision to publish the results.

\section{Abbreviations}

$\begin{array}{ll}\text { 2D:4D } & \text { Second-to-fourth finger length ratio } \\ \text { CDT } & \text { Carbohydrate-deficient transferrin } \\ \text { DSM-5 } & \text { Fifth edition of the Diagnostic and Statistical Manual of Mental Disorders } \\ \text { FASD } & \text { Fetal alcohol spectrum disorder } \\ \text { FDR } & \text { False discovery rate } \\ \text { ICD-10 } & \text { Tenth revision of the International Classification of Diseases } \\ \text { IQR } & \text { Interquartile range } \\ \text { TTT } & \text { Twin testosterone transfer model } \\ \text { WHO } & \text { World Health Organization }\end{array}$




\section{Appendix A}

Table A1. Spearman correlations of body measurements with 2D:4D in male and female alcohol-dependent in-patients (ADP) and healthy control subjects (HCS).

\begin{tabular}{|c|c|c|c|c|c|c|c|c|c|c|c|c|}
\hline & \multicolumn{6}{|c|}{ Males } & \multicolumn{6}{|c|}{ Females } \\
\hline & \multicolumn{3}{|c|}{ ADP } & \multicolumn{3}{|c|}{ HCS } & \multicolumn{3}{|c|}{ ADP } & \multicolumn{3}{|c|}{ HCS } \\
\hline & $N$ & $\rho$ & $P$ & $N$ & $\rho$ & $P$ & $N$ & $\rho$ & $P$ & $N$ & $\rho$ & $P$ \\
\hline \multicolumn{13}{|l|}{ Absolute measure } \\
\hline Body height & 103 & 0.049 & 0.625 & 133 & -0.068 & 0.439 & 76 & -0.016 & 0.894 & 105 & 0.090 & 0.360 \\
\hline Body weight & 102 & -0.112 & 0.261 & 133 & -0.059 & 0.501 & 76 & -0.104 & 0.369 & 104 & 0.006 & 0.955 \\
\hline Head circumference & 103 & 0.011 & 0.914 & 133 & -0.109 & 0.210 & 78 & 0.060 & 0.603 & 105 & -0.038 & 0.703 \\
\hline Bitragion head arc & 103 & 0.003 & 0.979 & 133 & 0.062 & 0.480 & 78 & 0.008 & 0.944 & 105 & -0.015 & 0.878 \\
\hline Sagittal head arc & 103 & 0.007 & 0.943 & 133 & -0.048 & 0.587 & 78 & 0.029 & 0.802 & 105 & -0.057 & 0.564 \\
\hline Mandibular arc & 102 & -0.066 & 0.512 & 133 & -0.056 & 0.526 & 76 & -0.130 & 0.264 & 104 & 0.112 & 0.259 \\
\hline Lip-chin distance & 102 & -0.021 & 0.832 & 132 & -0.034 & 0.702 & 78 & -0.069 & 0.549 & 105 & -0.092 & 0.351 \\
\hline Wrist circumference & 98 & -0.156 & 0.126 & 128 & -0.091 & 0.308 & 74 & 0.061 & 0.609 & 101 & -0.124 & 0.218 \\
\hline Waist circumference & 102 & -0.143 & 0.153 & 133 & -0.131 & 0.134 & 76 & -0.017 & 0.886 & 105 & 0.026 & 0.789 \\
\hline Hip circumference & 102 & -0.187 & 0.060 & 133 & -0.086 & 0.325 & 76 & -0.074 & 0.523 & 105 & -0.024 & 0.810 \\
\hline Thigh circumference & 102 & -0.052 & 0.601 & 133 & -0.086 & 0.323 & 78 & -0.119 & 0.297 & 104 & -0.059 & 0.549 \\
\hline Calf circumference & 102 & -0.082 & 0.412 & 132 & -0.046 & 0.599 & 78 & -0.055 & 0.635 & 105 & 0.065 & 0.508 \\
\hline Ankle circumference & 102 & -0.041 & 0.682 & 131 & -0.176 & 0.044 & 78 & 0.055 & 0.630 & 105 & 0.051 & 0.603 \\
\hline Foot length & 96 & 0.061 & 0.556 & 105 & 0.074 & 0.451 & 76 & 0.047 & 0.689 & 98 & 0.039 & 0.702 \\
\hline Foot breadth & 97 & -0.021 & 0.842 & 107 & -0.088 & 0.368 & 77 & 0.030 & 0.798 & 99 & -0.025 & 0.806 \\
\hline \multicolumn{13}{|l|}{ Measure divided by body height } \\
\hline Head circumference & 103 & -0.037 & 0.711 & 133 & -0.002 & 0.985 & 76 & 0.022 & 0.853 & 105 & -0.169 & 0.085 \\
\hline Bitragion head arc & 103 & -0.032 & 0.748 & 133 & 0.105 & 0.227 & 76 & 0.010 & 0.929 & 105 & -0.090 & 0.361 \\
\hline Sagittal head arc & 103 & 0.000 & 0.999 & 133 & -0.002 & 0.981 & 76 & 0.049 & 0.671 & 105 & -0.151 & 0.124 \\
\hline Mandibular arc & 102 & -0.092 & 0.358 & 133 & -0.040 & 0.649 & 74 & -0.105 & 0.372 & 104 & 0.016 & 0.875 \\
\hline Lip-chin distance & 102 & -0.057 & 0.570 & 132 & -0.027 & 0.762 & 76 & -0.056 & 0.634 & 105 & -0.130 & 0.188 \\
\hline Wrist circumference & 98 & -0.204 & 0.044 & 128 & -0.018 & 0.836 & 72 & 0.059 & 0.622 & 101 & -0.198 & 0.048 \\
\hline Waist circumference & 102 & -0.153 & 0.125 & 133 & -0.084 & 0.335 & 74 & -0.011 & 0.926 & 105 & -0.019 & 0.850 \\
\hline Hip circumference & 102 & -0.197 & 0.047 & 133 & -0.039 & 0.655 & 74 & -0.023 & 0.845 & 105 & -0.073 & 0.459 \\
\hline Thigh circumference & 102 & -0.060 & 0.549 & 133 & -0.043 & 0.626 & 76 & -0.093 & 0.425 & 104 & -0.122 & 0.217 \\
\hline Calf circumference & 102 & -0.079 & 0.431 & 132 & -0.031 & 0.726 & 76 & -0.004 & 0.975 & 105 & -0.003 & 0.979 \\
\hline Ankle circumference & 102 & -0.090 & 0.367 & 131 & -0.153 & 0.082 & 76 & 0.079 & 0.496 & 105 & -0.060 & 0.546 \\
\hline Foot length & 96 & -0.043 & 0.676 & 105 & 0.060 & 0.546 & 73 & 0.067 & 0.573 & 98 & -0.092 & 0.368 \\
\hline Foot breadth & 97 & -0.103 & 0.313 & 107 & -0.087 & 0.374 & 74 & 0.020 & 0.864 & 99 & -0.097 & 0.341 \\
\hline
\end{tabular}


Table A1. Cont.

\begin{tabular}{|c|c|c|c|c|c|c|c|c|c|c|c|c|}
\hline & \multicolumn{6}{|c|}{ Males } & \multicolumn{6}{|c|}{ Females } \\
\hline & \multicolumn{3}{|c|}{ ADP } & \multicolumn{3}{|c|}{ HCS } & \multicolumn{3}{|c|}{ ADP } & \multicolumn{3}{|c|}{ HCS } \\
\hline & $N$ & $\rho$ & $P$ & $N$ & $\rho$ & $P$ & $N$ & $\rho$ & $P$ & $N$ & $\rho$ & $P$ \\
\hline \multicolumn{13}{|c|}{ Measure residualized on body height } \\
\hline Head circumference & 103 & -0.003 & 0.979 & 133 & -0.077 & 0.378 & 76 & 0.043 & 0.715 & 105 & -0.107 & 0.276 \\
\hline Bitragion head arc & 103 & -0.006 & 0.952 & 133 & 0.082 & 0.349 & 76 & -0.019 & 0.872 & 105 & -0.042 & 0.674 \\
\hline Sagittal head arc & 103 & 0.017 & 0.868 & 133 & -0.018 & 0.840 & 76 & 0.002 & 0.988 & 105 & -0.095 & 0.335 \\
\hline Mandibular arc & 102 & -0.090 & 0.367 & 133 & -0.046 & 0.596 & 74 & -0.138 & 0.241 & 104 & 0.050 & 0.617 \\
\hline Lip-chin distance & 102 & -0.059 & 0.556 & 132 & -0.032 & 0.717 & 76 & -0.054 & 0.641 & 105 & -0.120 & 0.221 \\
\hline Wrist circumference & 98 & -0.204 & 0.044 & 128 & -0.021 & 0.810 & 72 & 0.059 & 0.624 & 101 & -0.201 & 0.044 \\
\hline Waist circumference & 102 & -0.156 & 0.118 & 133 & -0.101 & 0.245 & 74 & -0.018 & 0.882 & 105 & -0.014 & 0.887 \\
\hline Hip circumference & 102 & -0.190 & 0.055 & 133 & -0.086 & 0.326 & 74 & -0.062 & 0.602 & 105 & -0.026 & 0.789 \\
\hline Thigh circumference & 102 & -0.055 & 0.585 & 133 & -0.081 & 0.355 & 76 & -0.121 & 0.296 & 104 & -0.066 & 0.503 \\
\hline Calf circumference & 102 & -0.083 & 0.407 & 132 & -0.056 & 0.524 & 76 & -0.044 & 0.703 & 105 & 0.040 & 0.688 \\
\hline Ankle circumference & 102 & -0.092 & 0.357 & 131 & -0.180 & 0.040 & 76 & 0.069 & 0.556 & 105 & -0.027 & 0.787 \\
\hline Foot length & 96 & -0.048 & 0.641 & 105 & 0.051 & 0.606 & 73 & 0.077 & 0.519 & 98 & -0.095 & 0.354 \\
\hline Foot breadth & 97 & -0.091 & 0.377 & 107 & -0.091 & 0.351 & 74 & 0.000 & 0.999 & 99 & -0.098 & 0.333 \\
\hline
\end{tabular}

$P<0.05$ in bold. Significant negative correlation. 
Table A2. Spearman correlations of body measurements with the age of onset of regular alcohol drinking (i.e., daily over at least 7 days) and the age of first in-patient treatment due to alcohol problems in male and female alcohol-dependent in-patients.

\begin{tabular}{|c|c|c|c|c|c|c|c|c|c|c|c|c|}
\hline & \multicolumn{6}{|c|}{ Males: The Age of } & \multicolumn{6}{|c|}{ Females: The Age of } \\
\hline & \multicolumn{3}{|c|}{$\begin{array}{l}\text { Onset of Regular Alcohol Drinking (i.e., } \\
\text { Daily over at Least } 7 \text { Days) }\end{array}$} & \multicolumn{3}{|c|}{$\begin{array}{l}\text { First In-Patient Treatment } \\
\text { Due to Alcohol Problems }\end{array}$} & \multicolumn{3}{|c|}{$\begin{array}{l}\text { Onset of Regular Alcohol Drinking (i.e., } \\
\text { Daily over at Least } 7 \text { Days) }\end{array}$} & \multicolumn{3}{|c|}{$\begin{array}{l}\text { First In-Patient Treatment } \\
\text { Due to Alcohol Problems }\end{array}$} \\
\hline & $N$ & $\rho$ & $P$ & $N$ & $\rho$ & $P$ & $N$ & $\rho$ & $P$ & $N$ & $\rho$ & $P$ \\
\hline \multicolumn{13}{|l|}{ Absolute measure } \\
\hline Body height & 91 & -0.087 & 0.412 & 95 & -0.043 & 0.677 & 61 & -0.145 & 0.265 & 71 & -0.089 & 0.462 \\
\hline Body weight & 91 & -0.070 & 0.510 & 95 & 0.133 & 0.201 & 61 & 0.031 & 0.812 & 71 & 0.055 & 0.650 \\
\hline Head circumference & 91 & 0.039 & 0.715 & 95 & 0.129 & 0.212 & 63 & -0.015 & 0.906 & 73 & 0.030 & 0.800 \\
\hline Bitragion head arc & 91 & 0.127 & 0.231 & 95 & 0.110 & 0.288 & 63 & 0.061 & 0.637 & 73 & 0.159 & 0.180 \\
\hline Sagittal head arc & 91 & -0.225 & 0.032 & 95 & -0.311 & 0.002 & 63 & 0.056 & 0.662 & 73 & 0.116 & 0.330 \\
\hline Mandibular arc & 90 & 0.213 & 0.044 & 94 & 0.202 & 0.051 & 61 & 0.084 & 0.519 & 71 & 0.051 & 0.674 \\
\hline Lip-chin distance & 90 & 0.011 & 0.921 & 94 & 0.115 & 0.271 & 63 & 0.023 & 0.861 & 73 & 0.044 & 0.714 \\
\hline Wrist circumference & 87 & -0.077 & 0.478 & 91 & 0.150 & 0.157 & 61 & 0.132 & 0.311 & 70 & 0.202 & 0.094 \\
\hline Waist circumference & 91 & 0.016 & 0.880 & 95 & 0.215 & 0.036 & 63 & 0.124 & 0.331 & 71 & 0.110 & 0.359 \\
\hline Hip circumference & 91 & 0.061 & 0.569 & 95 & 0.221 & 0.031 & 63 & 0.024 & 0.851 & 71 & 0.111 & 0.356 \\
\hline Thigh circumference & 91 & -0.222 & 0.035 & 95 & 0.048 & 0.646 & 63 & -0.028 & 0.825 & 73 & 0.018 & 0.882 \\
\hline Calf circumference & 91 & -0.161 & 0.126 & 95 & 0.072 & 0.485 & 63 & 0.046 & 0.722 & 73 & 0.054 & 0.653 \\
\hline Ankle circumference & 91 & -0.103 & 0.333 & 95 & 0.234 & 0.022 & 63 & -0.093 & 0.470 & 73 & 0.108 & 0.361 \\
\hline Foot length & 84 & -0.097 & 0.382 & 87 & 0.090 & 0.405 & 62 & -0.123 & 0.339 & 71 & 0.025 & 0.838 \\
\hline Foot breadth & 85 & -0.049 & 0.659 & 88 & 0.155 & 0.149 & 63 & 0.189 & 0.137 & 72 & 0.180 & 0.130 \\
\hline \multicolumn{13}{|l|}{ Measure divided by body height } \\
\hline Head circumference & 91 & 0.097 & 0.361 & 95 & 0.078 & 0.451 & 61 & 0.170 & 0.190 & 71 & 0.120 & 0.319 \\
\hline Bitragion head arc & 91 & 0.164 & 0.121 & 95 & 0.136 & 0.190 & 61 & 0.219 & 0.090 & 71 & 0.219 & 0.067 \\
\hline Sagittal head arc & 91 & -0.147 & 0.164 & 95 & -0.266 & 0.009 & 61 & 0.187 & 0.149 & 71 & 0.193 & 0.107 \\
\hline Mandibular arc & 90 & 0.227 & 0.031 & 94 & 0.206 & 0.047 & 59 & 0.165 & 0.211 & 69 & 0.192 & 0.114 \\
\hline Lip-chin distance & 90 & 0.043 & 0.685 & 94 & 0.129 & 0.216 & 61 & 0.067 & 0.610 & 71 & 0.099 & 0.413 \\
\hline Wrist circumference & 87 & -0.087 & 0.424 & 91 & 0.156 & 0.140 & 59 & 0.271 & 0.038 & 68 & 0.339 & 0.005 \\
\hline Waist circumference & 91 & 0.004 & 0.973 & 95 & 0.216 & 0.035 & 61 & 0.200 & 0.122 & 69 & 0.208 & 0.087 \\
\hline Hip circumference & 91 & 0.074 & 0.486 & 95 & 0.195 & 0.058 & 61 & 0.108 & 0.409 & 69 & 0.201 & 0.098 \\
\hline Thigh circumference & 91 & -0.218 & 0.038 & 95 & 0.042 & 0.684 & 61 & 0.108 & 0.406 & 71 & 0.110 & 0.359 \\
\hline Calf circumference & 91 & -0.143 & 0.176 & 95 & 0.073 & 0.482 & 61 & 0.133 & 0.308 & 71 & 0.119 & 0.324 \\
\hline Ankle circumference & 91 & -0.057 & 0.590 & 95 & 0.226 & 0.028 & 61 & 0.055 & 0.671 & 71 & 0.287 & 0.015 \\
\hline Foot length & 84 & -0.009 & 0.939 & 87 & 0.174 & 0.107 & 59 & 0.003 & 0.985 & 68 & 0.300 & 0.013 \\
\hline Foot breadth & 85 & -0.024 & 0.826 & 88 & 0.149 & 0.166 & 60 & 0.321 & 0.012 & 69 & 0.287 & 0.017 \\
\hline
\end{tabular}


Table A2. Cont.

\begin{tabular}{|c|c|c|c|c|c|c|c|c|c|c|c|c|}
\hline & \multicolumn{6}{|c|}{ Males: The Age of } & \multicolumn{6}{|c|}{ Females: The Age of } \\
\hline & \multicolumn{3}{|c|}{$\begin{array}{c}\text { Onset of Regular Alcohol Drinking (i.e., } \\
\text { Daily over at Least } 7 \text { Days) }\end{array}$} & \multicolumn{3}{|c|}{$\begin{array}{l}\text { First In-Patient Treatment } \\
\text { Due to Alcohol Problems }\end{array}$} & \multicolumn{3}{|c|}{$\begin{array}{l}\text { Onset of Regular Alcohol Drinking (i.e., } \\
\text { Daily over at Least } 7 \text { Days) }\end{array}$} & \multicolumn{3}{|c|}{$\begin{array}{l}\text { First In-Patient Treatment } \\
\text { Due to Alcohol Problems }\end{array}$} \\
\hline & $N$ & $\rho$ & $P$ & $N$ & $\rho$ & $P$ & $N$ & $\rho$ & $P$ & $N$ & $\rho$ & $P$ \\
\hline Head circumference & 91 & 0.042 & 0.694 & 95 & 0.104 & 0.317 & 61 & 0.085 & 0.516 & 71 & 0.130 & 0.279 \\
\hline Bitragion head arc & 91 & 0.159 & 0.133 & 95 & 0.127 & 0.219 & 61 & 0.140 & 0.280 & 71 & 0.234 & 0.050 \\
\hline Sagittal head arc & 91 & -0.200 & 0.058 & 95 & -0.312 & 0.002 & 61 & 0.097 & 0.457 & 71 & 0.155 & 0.197 \\
\hline Mandibular arc & 90 & 0.244 & 0.020 & 94 & 0.209 & 0.043 & 59 & 0.155 & 0.242 & 69 & 0.158 & 0.194 \\
\hline Lip-chin distance & 90 & 0.039 & 0.718 & 94 & 0.128 & 0.219 & 61 & 0.058 & 0.654 & 71 & 0.084 & 0.487 \\
\hline Waist circumference & 91 & -0.012 & 0.907 & 95 & 0.207 & 0.045 & 61 & 0.198 & 0.126 & 69 & 0.192 & 0.114 \\
\hline Hip circumference & 91 & 0.069 & 0.518 & 95 & 0.222 & 0.030 & 61 & 0.055 & 0.674 & 69 & 0.141 & 0.246 \\
\hline Thigh circumference & 91 & -0.216 & 0.040 & 95 & 0.054 & 0.602 & 61 & 0.021 & 0.872 & 71 & 0.070 & 0.561 \\
\hline Calf circumference & 91 & -0.144 & 0.173 & 95 & 0.083 & 0.425 & 61 & 0.098 & 0.453 & 71 & 0.100 & 0.406 \\
\hline Ankle circumference & 91 & -0.070 & 0.507 & 95 & 0.229 & 0.026 & 61 & 0.019 & 0.886 & 71 & 0.260 & 0.028 \\
\hline Foot length & 84 & -0.012 & 0.915 & 87 & 0.177 & 0.101 & 59 & 0.014 & 0.918 & 68 & 0.319 & 0.008 \\
\hline Foot breadth & 85 & -0.040 & 0.713 & 88 & 0.149 & 0.166 & 60 & 0.302 & 0.019 & 69 & 0.287 & 0.017 \\
\hline
\end{tabular}

$P<0.05$ in bold. Significant positive correlation, significant negative correlation. 
Table A3. Spearman correlations of body measurements with total lifetime drinking and daily ethanol intake in male and female alcohol-dependent in-patients.

\begin{tabular}{|c|c|c|c|c|c|c|c|c|c|c|c|c|}
\hline & \multicolumn{6}{|c|}{ Males } & \multicolumn{6}{|c|}{ Females } \\
\hline & \multicolumn{3}{|c|}{ Total Lifetime Drinking } & \multicolumn{3}{|c|}{ Daily Ethanol Intake } & \multicolumn{3}{|c|}{ Total Lifetime Drinking } & \multicolumn{3}{|c|}{ Daily Ethanol Intake } \\
\hline & $N$ & $\rho$ & $P$ & $N$ & $\rho$ & $P$ & $N$ & $\rho$ & $P$ & $N$ & $\rho$ & $P$ \\
\hline \multicolumn{13}{|l|}{ Absolute measure } \\
\hline $\begin{array}{l}\text { Body height } \\
\end{array}$ & 87 & 0.001 & 0.993 & 85 & 0.142 & 0.196 & 57 & -0.051 & 0.705 & 55 & 0.060 & 0.664 \\
\hline Body weight & 87 & 0.073 & 0.504 & 85 & 0.099 & 0.368 & 57 & 0.188 & 0.162 & 55 & 0.075 & 0.587 \\
\hline Head circumference & 87 & -0.074 & 0.493 & 85 & -0.051 & 0.643 & 59 & -0.046 & 0.728 & 57 & 0.059 & 0.660 \\
\hline Bitragion head arc & 87 & 0.061 & 0.575 & 85 & 0.059 & 0.593 & 59 & 0.056 & 0.674 & 57 & 0.026 & 0.847 \\
\hline Sagittal head arc & 87 & -0.071 & 0.513 & 85 & 0.064 & 0.561 & 59 & 0.198 & 0.134 & 57 & 0.128 & 0.341 \\
\hline Mandibular arc & 86 & -0.144 & 0.186 & 84 & -0.189 & 0.085 & 57 & -0.115 & 0.393 & 55 & -0.272 & 0.045 \\
\hline Lip-chin distance & 86 & -0.228 & 0.035 & 84 & -0.188 & 0.086 & 59 & -0.188 & 0.155 & 57 & -0.080 & 0.553 \\
\hline Wrist circumference & 83 & 0.018 & 0.870 & 81 & 0.068 & 0.546 & 58 & 0.042 & 0.756 & 56 & -0.039 & 0.773 \\
\hline Waist circumference & 87 & 0.097 & 0.370 & 85 & 0.005 & 0.963 & 58 & 0.219 & 0.099 & 56 & 0.134 & 0.323 \\
\hline Hip circumference & 87 & 0.095 & 0.383 & 85 & 0.072 & 0.512 & 58 & 0.108 & 0.422 & 56 & 0.006 & 0.963 \\
\hline Thigh circumference & 87 & -0.035 & 0.745 & 85 & 0.040 & 0.718 & 59 & 0.066 & 0.618 & 57 & 0.103 & 0.444 \\
\hline Calf circumference & 87 & -0.019 & 0.860 & 85 & 0.073 & 0.506 & 59 & 0.047 & 0.721 & 57 & -0.059 & 0.661 \\
\hline Ankle circumference & 87 & -0.150 & 0.166 & 85 & -0.044 & 0.692 & 59 & 0.084 & 0.526 & 57 & -0.064 & 0.638 \\
\hline Foot length & 79 & -0.087 & 0.444 & 77 & 0.108 & 0.348 & 57 & 0.144 & 0.284 & 55 & 0.141 & 0.306 \\
\hline Foot breadth & 80 & -0.028 & 0.808 & 78 & -0.006 & 0.960 & 58 & 0.052 & 0.701 & 56 & -0.015 & 0.914 \\
\hline \multicolumn{13}{|l|}{ Measure divided by body height } \\
\hline Head circumference & 87 & -0.058 & 0.595 & 85 & -0.185 & 0.091 & 57 & -0.046 & 0.733 & 55 & -0.038 & 0.783 \\
\hline Bitragion head arc & 87 & 0.099 & 0.360 & 85 & -0.054 & 0.621 & 57 & 0.075 & 0.577 & 55 & -0.009 & 0.951 \\
\hline Sagittal head arc & 87 & -0.073 & 0.501 & 85 & -0.036 & 0.741 & 57 & 0.156 & 0.248 & 55 & 0.070 & 0.614 \\
\hline Mandibular arc & 86 & -0.154 & 0.156 & 84 & -0.242 & 0.027 & 55 & -0.129 & 0.349 & 53 & -0.301 & 0.029 \\
\hline Lip-chin distance & 86 & -0.204 & 0.059 & 84 & -0.211 & 0.054 & 57 & -0.201 & 0.134 & 55 & -0.111 & 0.419 \\
\hline Wrist circumference & 83 & 0.046 & 0.681 & 81 & -0.020 & 0.862 & 56 & 0.036 & 0.794 & 54 & -0.098 & 0.482 \\
\hline Waist circumference & 87 & 0.114 & 0.295 & 85 & -0.012 & 0.913 & 56 & 0.182 & 0.180 & 54 & 0.093 & 0.505 \\
\hline Hip circumference & 87 & 0.129 & 0.233 & 85 & 0.041 & 0.707 & 56 & 0.110 & 0.419 & 54 & -0.032 & 0.819 \\
\hline Thigh circumference & 87 & 0.010 & 0.924 & 85 & 0.017 & 0.874 & 57 & 0.000 & 0.997 & 55 & 0.033 & 0.812 \\
\hline Calf circumference & 87 & 0.000 & 0.997 & 85 & 0.046 & 0.677 & 57 & 0.055 & 0.686 & 55 & -0.117 & 0.394 \\
\hline Ankle circumference & 87 & -0.140 & 0.196 & 85 & -0.130 & 0.235 & 57 & 0.092 & 0.495 & 55 & -0.127 & 0.354 \\
\hline Foot length & 79 & -0.068 & 0.549 & 77 & 0.021 & 0.857 & 54 & 0.132 & 0.341 & 52 & 0.126 & 0.375 \\
\hline Foot breadth & 80 & 0.039 & 0.734 & 78 & -0.093 & 0.417 & 55 & 0.044 & 0.751 & 53 & -0.117 & 0.406 \\
\hline
\end{tabular}


Table A3. Cont.

\begin{tabular}{|c|c|c|c|c|c|c|c|c|c|c|c|c|}
\hline & \multicolumn{6}{|c|}{ Males } & \multicolumn{6}{|c|}{ Females } \\
\hline & \multicolumn{3}{|c|}{ Total Lifetime Drinking } & \multicolumn{3}{|c|}{ Daily Ethanol Intake } & \multicolumn{3}{|c|}{ Total Lifetime Drinking } & \multicolumn{3}{|c|}{ Daily Ethanol Intake } \\
\hline & $N$ & $\rho$ & $P$ & $N$ & $\rho$ & $P$ & $N$ & $\rho$ & $P$ & $N$ & $\rho$ & $P$ \\
\hline Head circumference & 87 & -0.077 & 0.477 & 85 & -0.120 & 0.275 & 57 & -0.080 & 0.556 & 55 & 0.018 & 0.898 \\
\hline Bitragion head arc & 87 & 0.069 & 0.525 & 85 & 0.009 & 0.934 & 57 & 0.058 & 0.666 & 55 & 0.003 & 0.980 \\
\hline Sagittal head arc & 87 & -0.072 & 0.509 & 85 & 0.007 & 0.946 & 57 & 0.197 & 0.142 & 55 & 0.135 & 0.327 \\
\hline Mandibular arc & 86 & -0.151 & 0.166 & 84 & -0.226 & 0.039 & 55 & -0.154 & 0.261 & 53 & -0.306 & 0.026 \\
\hline Lip-chin distance & 86 & -0.207 & 0.056 & 84 & -0.189 & 0.085 & 57 & -0.209 & 0.119 & 55 & -0.116 & 0.400 \\
\hline Wrist circumference & 83 & 0.035 & 0.752 & 81 & -0.013 & 0.908 & 56 & 0.037 & 0.784 & 54 & -0.094 & 0.501 \\
\hline Waist circumference & 87 & 0.115 & 0.290 & 85 & -0.009 & 0.936 & 56 & 0.193 & 0.154 & 54 & 0.119 & 0.393 \\
\hline Hip circumference & 87 & 0.099 & 0.361 & 85 & 0.067 & 0.545 & 56 & 0.090 & 0.511 & 54 & 0.008 & 0.954 \\
\hline Thigh circumference & 87 & -0.025 & 0.822 & 85 & 0.038 & 0.731 & 57 & 0.037 & 0.784 & 55 & 0.091 & 0.508 \\
\hline Calf circumference & 87 & -0.003 & 0.979 & 85 & 0.062 & 0.573 & 57 & 0.052 & 0.700 & 55 & -0.079 & 0.566 \\
\hline Ankle circumference & 87 & -0.158 & 0.144 & 85 & -0.109 & 0.320 & 57 & 0.097 & 0.473 & 55 & -0.086 & 0.534 \\
\hline Foot length & 79 & -0.068 & 0.554 & 77 & 0.020 & 0.862 & 54 & 0.133 & 0.339 & 52 & 0.111 & 0.434 \\
\hline Foot breadth & 80 & 0.046 & 0.684 & 78 & -0.067 & 0.562 & 55 & 0.032 & 0.814 & 53 & -0.107 & 0.446 \\
\hline
\end{tabular}


Table A4. Inter-correlations between absolute male body measurements (alcohol-dependent in-patients bottom left, healthy control subjects top right).

\begin{tabular}{|c|c|c|c|c|c|c|c|c|c|c|c|c|c|c|c|}
\hline$N$ & & 133 & 133 & 133 & 133 & 133 & 132 & 128 & 133 & 133 & 133 & 132 & 131 & 105 & 107 \\
\hline$\rho$ & Body & 0.264 & 0.227 & 0.232 & 0.295 & 0.141 & 0.146 & 0.189 & -0.097 & 0.090 & 0.029 & 0.065 & 0.335 & 0.610 & 0.474 \\
\hline$P$ & & 0.002 & 0.009 & 0.007 & 0.001 & 0.105 & 0.095 & 0.033 & 0.269 & 0.303 & 0.741 & 0.461 & $<0.001$ & $<0.001$ & $<0.001$ \\
\hline$N$ & 106 & & 133 & 133 & 133 & 133 & 132 & 128 & 133 & 133 & 133 & 132 & 131 & 105 & 107 \\
\hline$\rho$ & 0.368 & Body & 0.259 & 0.074 & 0.026 & 0.204 & 0.166 & 0.626 & 0.818 & 0.874 & 0.698 & 0.675 & 0.467 & 0.213 & 0.360 \\
\hline$P$ & $<0.001$ & & 0.003 & 0.395 & 0.765 & 0.019 & 0.057 & $<0.001$ & $<0.001$ & $<0.001$ & $<0.001$ & $<0.001$ & $<0.001$ & 0.029 & $<0.001$ \\
\hline$N$ & 107 & 106 & & 133 & 133 & 133 & 132 & 128 & 133 & 133 & 133 & 132 & 131 & 105 & 107 \\
\hline$\rho$ & 0.175 & 0.453 & Head & 0.500 & 0.475 & 0.152 & 0.271 & 0.319 & 0.183 & 0.229 & 0.206 & 0.304 & 0.408 & 0.338 & 0.264 \\
\hline$P$ & 0.071 & $<0.001$ & circum-ference & $<0.001$ & $<0.001$ & 0.081 & 0.002 & $<0.001$ & 0.035 & 0.008 & 0.017 & $<0.001$ & $<0.001$ & $<0.001$ & 0.006 \\
\hline$N$ & 107 & 106 & 107 & Bitragion & 133 & 133 & 132 & 128 & 133 & 133 & 133 & 132 & 131 & 105 & 107 \\
\hline$\rho$ & 0.326 & 0.168 & 0.390 & head & 0.393 & -0.013 & 0.138 & 0.143 & -0.004 & 0.044 & 0.038 & 0.046 & 0.198 & 0.351 & 0.215 \\
\hline$P$ & 0.001 & 0.085 & $<0.001$ & arc & $<0.001$ & 0.880 & 0.115 & 0.107 & 0.961 & 0.612 & 0.661 & 0.600 & 0.023 & $<0.001$ & 0.026 \\
\hline$N$ & 107 & 106 & 107 & 107 & Sagittal & 133 & 132 & 128 & 133 & 133 & 133 & 132 & 131 & 105 & 107 \\
\hline$\rho$ & 0.285 & 0.031 & 0.288 & 0.341 & head & 0.040 & 0.162 & 0.020 & -0.094 & 0.011 & 0.081 & 0.120 & 0.248 & 0.219 & 0.111 \\
\hline$P$ & 0.003 & 0.749 & 0.003 & $<0.001$ & arc & 0.647 & 0.063 & 0.819 & 0.281 & 0.901 & 0.353 & 0.171 & 0.004 & 0.025 & 0.255 \\
\hline N & 106 & 105 & 106 & 106 & 106 & & 132 & 128 & 133 & 133 & 133 & 132 & 131 & 105 & 107 \\
\hline$\rho$ & 0.115 & 0.411 & 0.283 & 0.161 & -0.085 & Mandi-bular & 0.330 & 0.303 & 0.195 & 0.162 & 0.089 & 0.245 & 0.348 & 0.213 & 0.128 \\
\hline$P$ & 0.239 & $<0.001$ & 0.003 & 0.099 & 0.389 & arc & $<0.001$ & 0.001 & 0.024 & 0.062 & 0.306 & 0.005 & $<0.001$ & 0.029 & 0.188 \\
\hline N & 106 & 105 & 106 & 106 & 106 & 105 & & 127 & 132 & 132 & 132 & 131 & 130 & 104 & 106 \\
\hline$\rho$ & 0.001 & 0.187 & 0.284 & 0.094 & -0.259 & 0.397 & Lip-chin & 0.239 & 0.127 & 0.140 & 0.190 & 0.229 & 0.201 & 0.282 & 0.229 \\
\hline$P$ & 0.990 & 0.055 & 0.003 & 0.339 & 0.007 & $<0.001$ & distance & 0.007 & 0.147 & 0.110 & 0.029 & 0.008 & 0.022 & 0.004 & 0.018 \\
\hline$N$ & 102 & 101 & 102 & 102 & 102 & 101 & 101 & & 128 & 128 & 128 & 127 & 126 & 101 & 103 \\
\hline$\rho$ & 0.337 & 0.692 & 0.414 & 0.222 & 0.001 & 0.467 & 0.337 & Wrist & 0.503 & 0.552 & 0.459 & 0.483 & 0.535 & 0.187 & 0.361 \\
\hline$P$ & 0.001 & $<0.001$ & $<0.001$ & 0.025 & 0.995 & $<0.001$ & 0.001 & circum-ference & $<0.001$ & $<0.001$ & $<0.001$ & $<0.001$ & $<0.001$ & 0.061 & $<0.001$ \\
\hline N & 106 & 105 & 106 & 106 & 106 & 105 & 105 & 102 & & 133 & 133 & 132 & 131 & 105 & 107 \\
\hline$\rho$ & 0.169 & 0.886 & 0.395 & 0.183 & -0.081 & 0.455 & 0.259 & 0.569 & Waist & 0.778 & 0.581 & 0.531 & 0.314 & 0.013 & 0.135 \\
\hline$P$ & 0.084 & $<0.001$ & $<0.001$ & 0.061 & 0.407 & $<0.001$ & 0.008 & $<0.001$ & circum-ference & $<0.001$ & $<0.001$ & $<0.001$ & $<0.001$ & 0.893 & 0.165 \\
\hline$N$ & 106 & 105 & 106 & 106 & 106 & 105 & 105 & 102 & 106 & & 133 & 132 & 131 & 105 & 107 \\
\hline$\rho$ & 0.263 & 0.878 & 0.457 & 0.228 & -0.012 & 0.435 & 0.242 & 0.623 & 0.839 & Hip & 0.768 & 0.625 & 0.377 & 0.088 & 0.229 \\
\hline$P$ & 0.007 & $<0.001$ & $<0.001$ & 0.019 & 0.905 & $<0.001$ & 0.013 & $<0.001$ & $<0.001$ & circum-ference & $<0.001$ & $<0.001$ & $<0.001$ & 0.371 & 0.018 \\
\hline N & 106 & 105 & 106 & 106 & 106 & 105 & 105 & 102 & 106 & 106 & & 132 & 131 & 105 & 107 \\
\hline$\rho$ & 0.160 & 0.831 & 0.426 & 0.012 & -0.033 & 0.428 & 0.291 & 0.595 & 0.725 & 0.708 & Thigh & 0.673 & 0.319 & -0.067 & 0.157 \\
\hline$P$ & 0.101 & $<0.001$ & $<0.001$ & 0.902 & 0.739 & $<0.001$ & 0.003 & $<0.001$ & $<0.001$ & $<0.001$ & & $<0.001$ & $<0.001$ & 0.495 & 0.107 \\
\hline N & 106 & 105 & 106 & 106 & 106 & 105 & 105 & 102 & 106 & 106 & 106 & & 131 & 104 & 106 \\
\hline$\rho$ & 0.126 & 0.724 & 0.285 & 0.077 & 0.024 & 0.336 & 0.155 & 0.560 & 0.580 & 0.569 & 0.706 & Calf & 0.417 & 0.054 & 0.348 \\
\hline$P$ & 0.199 & $<0.001$ & 0.003 & 0.430 & 0.807 & $<0.001$ & 0.115 & $<0.001$ & $<0.001$ & $<0.001$ & $<0.001$ & circum-ference & $<0.001$ & 0.587 & $<0.001$ \\
\hline N & 106 & 105 & 106 & 106 & 106 & 105 & 105 & 102 & 106 & 106 & 106 & 106 & & 103 & 105 \\
\hline$\rho$ & 0.323 & 0.626 & 0.424 & 0.264 & -0.060 & 0.347 & 0.381 & 0.580 & 0.574 & 0.578 & 0.539 & 0.582 & Ankle & 0.497 & 0.565 \\
\hline$P$ & 0.001 & $<0.001$ & $<0.001$ & 0.006 & 0.543 & $<0.001$ & $<0.001$ & $<0.001$ & $<0.001$ & $<0.001$ & $<0.001$ & $<0.001$ & circum-ference & $<0.001$ & $<0.001$ \\
\hline N & 99 & 98 & 99 & 99 & 99 & 98 & 98 & 95 & 98 & 98 & 98 & 98 & 98 & & 105 \\
\hline$\rho$ & 0.690 & 0.497 & 0.253 & 0.293 & 0.239 & 0.245 & 0.262 & 0.480 & 0.354 & 0.425 & 0.354 & 0.330 & 0.544 & Foot & 0.527 \\
\hline$P$ & $<0.001$ & $<0.001$ & 0.012 & 0.003 & 0.017 & 0.015 & 0.009 & $<0.001$ & $<0.001$ & $<0.001$ & $<0.001$ & 0.001 & $<0.001$ & & $<0.001$ \\
\hline$N$ & 100 & 99 & 100 & 100 & 100 & 99 & 99 & 95 & 99 & 99 & 99 & 99 & 99 & 99 & \\
\hline$\rho$ & 0.293 & 0.563 & 0.368 & 0.234 & -0.034 & 0.264 & 0.253 & 0.602 & 0.443 & 0.482 & 0.431 & 0.472 & 0.636 & 0.494 & Foot \\
\hline$P$ & 0.003 & $<0.001$ & $<0.001$ & 0.019 & 0.739 & 0.008 & 0.012 & $<0.001$ & $<0.001$ & $<0.001$ & $<0.001$ & $<0.001$ & $<0.001$ & $<0.001$ & breadth \\
\hline
\end{tabular}


Table A5. Inter-correlations between absolute female body measurements (alcohol-dependent in-patients bottom left, healthy control subjects top right).

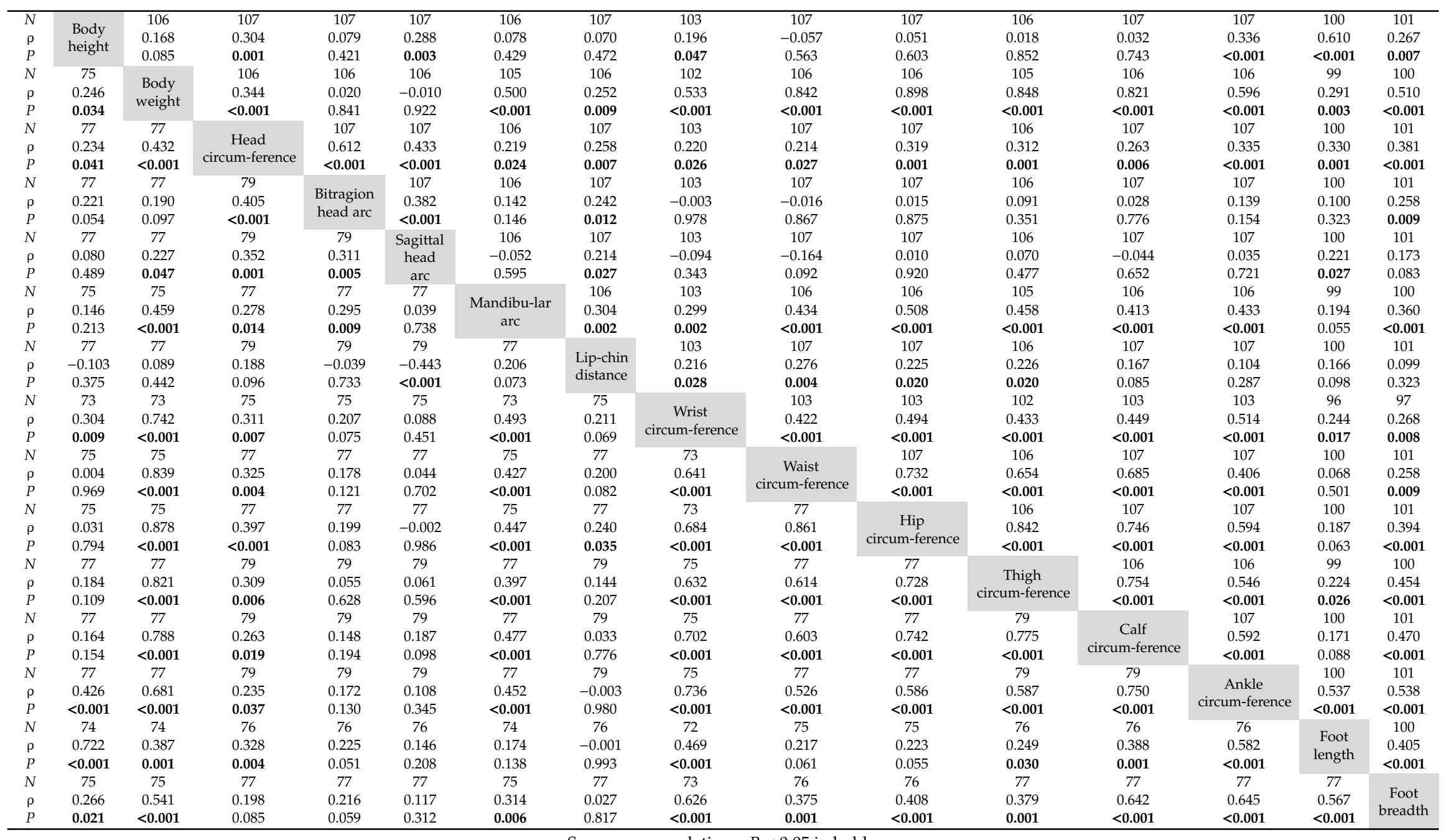

Spearman correlations. $P<0.05$ in bold. 
Table A6. Inter-correlations between male body measurements divided by height (alcohol-dependent in-patients bottom left, healthy control subjects top right).

\begin{tabular}{|c|c|c|c|c|c|c|c|c|c|c|c|c|c|}
\hline $\bar{N}$ & & 133 & 133 & 133 & 132 & 128 & 133 & 133 & 133 & 132 & 131 & 105 & 107 \\
\hline$\rho$ & $\begin{array}{l}\text { Head } \\
\text {. }\end{array}$ & 0.642 & 0.488 & 0.253 & 0.299 & 0.497 & 0.431 & 0.449 & 0.421 & 0.511 & 0.502 & 0.321 & 0.383 \\
\hline$P$ & circum-ference & $<0.001$ & $<0.001$ & 0.003 & $<0.001$ & $<0.001$ & $<0.001$ & $<0.001$ & $<0.001$ & $<0.001$ & $<0.001$ & 0.001 & $<0.001$ \\
\hline$N$ & 107 & & 133 & 133 & 132 & 128 & 133 & 133 & 133 & 132 & 131 & 105 & 107 \\
\hline$\rho$ & 0.547 & Bitragion & 0.486 & 0.085 & 0.172 & 0.297 & 0.227 & 0.237 & 0.230 & 0.229 & 0.336 & 0.280 & 0.255 \\
\hline$P$ & $<0.001$ & head arc & $<0.001$ & 0.333 & 0.049 & 0.001 & 0.009 & 0.006 & 0.008 & 0.008 & $<0.001$ & 0.004 & 0.008 \\
\hline$N$ & 107 & 107 & \multirow{3}{*}{$\begin{array}{l}\text { Sagittal } \\
\text { head arc }\end{array}$} & 133 & 132 & 128 & 133 & 133 & 133 & 132 & 131 & 105 & 107 \\
\hline$\rho$ & 0.459 & 0.374 & & 0.064 & 0.196 & 0.156 & 0.101 & 0.162 & 0.222 & 0.232 & 0.246 & 0.080 & 0.084 \\
\hline$P$ & $<0.001$ & $<0.001$ & & 0.467 & 0.024 & 0.079 & 0.246 & 0.063 & 0.010 & 0.007 & 0.005 & 0.416 & 0.390 \\
\hline$N$ & 106 & 106 & 106 & \multirow{3}{*}{$\begin{array}{l}\text { Mandibular } \\
\text { arc }\end{array}$} & 132 & 128 & 133 & 133 & 133 & 132 & 131 & 105 & 107 \\
\hline$\rho$ & 0.390 & 0.183 & -0.004 & & 0.338 & 0.397 & 0.297 & 0.284 & 0.194 & 0.362 & 0.374 & 0.208 & 0.151 \\
\hline$P$ & $<0.001$ & 0.061 & 0.964 & & $<0.001$ & $<0.001$ & 0.001 & 0.001 & 0.025 & $<0.001$ & $<0.001$ & 0.033 & 0.121 \\
\hline$N$ & 106 & 106 & 106 & 105 & \multirow{3}{*}{$\begin{array}{l}\text { Lip-chin } \\
\text { distance }\end{array}$} & 127 & 132 & 132 & 132 & 131 & 130 & 104 & 106 \\
\hline$\rho$ & 0.360 & 0.198 & -0.147 & 0.434 & & 0.253 & 0.217 & 0.224 & 0.281 & 0.298 & 0.241 & 0.242 & 0.141 \\
\hline$P$ & $<0.001$ & 0.042 & 0.132 & $<0.001$ & & 0.004 & 0.012 & 0.010 & 0.001 & 0.001 & 0.006 & 0.013 & 0.149 \\
\hline$N$ & 102 & 102 & 102 & 101 & 101 & \multirow{3}{*}{$\begin{array}{l}\text { Wrist } \\
\text { circum-ference }\end{array}$} & 128 & 128 & 128 & 127 & 126 & 101 & 103 \\
\hline$\rho$ & 0.388 & 0.195 & -0.039 & 0.449 & 0.378 & & 0.602 & 0.644 & 0.510 & 0.590 & 0.601 & 0.271 & 0.404 \\
\hline$P$ & $<0.001$ & 0.050 & 0.694 & $<0.001$ & $<0.001$ & & $<0.001$ & $<0.001$ & $<0.001$ & $<0.001$ & $<0.001$ & 0.006 & $<0.001$ \\
\hline$N$ & 106 & 106 & 106 & 105 & 105 & 102 & \multirow{3}{*}{$\begin{array}{c}\text { Waist } \\
\text { circum-ference }\end{array}$} & 133 & 133 & 132 & 131 & 105 & 107 \\
\hline$\rho$ & 0.350 & 0.198 & -0.085 & 0.402 & 0.275 & 0.559 & & 0.810 & 0.633 & 0.623 & 0.489 & 0.181 & 0.352 \\
\hline$P$ & $<0.001$ & 0.041 & 0.388 & $<0.001$ & 0.004 & $<0.001$ & & $<0.001$ & $<0.001$ & $<0.001$ & $<0.001$ & 0.065 & $<0.001$ \\
\hline N & 106 & 106 & 106 & 105 & 105 & 102 & 106 & \multirow{3}{*}{$\begin{array}{c}\text { Hip } \\
\text { circum-ference }\end{array}$} & 133 & 132 & 131 & 105 & 107 \\
\hline$\rho$ & 0.366 & 0.199 & -0.047 & 0.408 & 0.283 & 0.573 & 0.842 & & 0.783 & 0.694 & 0.519 & 0.142 & 0.348 \\
\hline$P$ & $<0.001$ & 0.041 & 0.631 & $<0.001$ & 0.003 & $<0.001$ & $<0.001$ & & $<0.001$ & $<0.001$ & $<0.001$ & 0.149 & $<0.001$ \\
\hline$N$ & 106 & 106 & 106 & 105 & 105 & 102 & 106 & 106 & \multirow{3}{*}{$\begin{array}{l}\text { Thigh } \\
\text { circum-ference }\end{array}$} & 132 & 131 & 105 & 107 \\
\hline$\rho$ & 0.398 & 0.019 & 0.004 & 0.416 & 0.290 & 0.575 & 0.712 & 0.709 & & 0.714 & 0.452 & -0.059 & 0.263 \\
\hline$P$ & $<0.001$ & 0.849 & 0.971 & $<0.001$ & 0.003 & $<0.001$ & $<0.001$ & $<0.001$ & & $<0.001$ & $<0.001$ & 0.547 & 0.006 \\
\hline$N$ & 106 & 106 & 106 & 105 & 105 & 102 & 106 & 106 & 106 & \multirow{3}{*}{$\begin{array}{c}\text { Calf } \\
\text { circum-ference }\end{array}$} & 131 & 104 & 106 \\
\hline$\rho$ & 0.330 & 0.158 & 0.076 & 0.322 & 0.182 & 0.598 & 0.625 & 0.586 & 0.695 & & 0.533 & 0.088 & 0.439 \\
\hline$P$ & 0.001 & 0.107 & 0.439 & 0.001 & 0.062 & $<0.001$ & $<0.001$ & $<0.001$ & $<0.001$ & & $<0.001$ & 0.376 & $<0.001$ \\
\hline$N$ & 106 & 106 & 106 & 105 & 105 & 102 & 106 & 106 & 106 & 106 & \multirow{3}{*}{$\begin{array}{l}\text { Ankle } \\
\text { circum-ference }\end{array}$} & 103 & 105 \\
\hline$\rho$ & 0.434 & 0.260 & -0.045 & 0.444 & 0.452 & 0.584 & 0.532 & 0.544 & 0.534 & 0.631 & & 0.399 & 0.526 \\
\hline$P$ & $<0.001$ & 0.007 & 0.644 & $<0.001$ & $<0.001$ & $<0.001$ & $<0.001$ & $<0.001$ & $<0.001$ & $<0.001$ & & $<0.001$ & $<0.001$ \\
\hline$N$ & 99 & 99 & 99 & 98 & 98 & 95 & 98 & 98 & 98 & 98 & 98 & & 105 \\
\hline$\rho$ & 0.196 & 0.140 & 0.068 & 0.332 & 0.373 & 0.370 & 0.312 & 0.354 & 0.290 & 0.296 & 0.523 & Foot & 0.356 \\
\hline$P$ & 0.052 & 0.166 & 0.505 & 0.001 & $<0.001$ & $<0.001$ & 0.002 & $<0.001$ & 0.004 & 0.003 & $<0.001$ & length & $<0.001$ \\
\hline$N$ & 100 & 100 & 100 & 99 & 99 & 95 & 99 & 99 & 99 & 99 & 99 & 99 & \multirow{3}{*}{$\begin{array}{c}\text { Foot } \\
\text { breadtt }\end{array}$} \\
\hline$\rho$ & 0.411 & 0.282 & 0.040 & 0.271 & 0.292 & 0.538 & 0.351 & 0.403 & 0.373 & 0.472 & 0.629 & 0.436 & \\
\hline$P$ & $<0.001$ & 0.004 & 0.695 & 0.007 & 0.003 & $<0.001$ & $<0.001$ & $<0.001$ & $<0.001$ & $<0.001$ & $<0.001$ & $<0.001$ & \\
\hline
\end{tabular}


Table A7. Inter-correlations between female body measurements divided by height (alcohol-dependent in-patients bottom left, healthy control subjects top right).

\begin{tabular}{|c|c|c|c|c|c|c|c|c|c|c|c|c|c|}
\hline$N$ & & 107 & 107 & 106 & 107 & 103 & 107 & 107 & 106 & 107 & 107 & 100 & 101 \\
\hline$\rho$ & $\begin{array}{l}\text { Head } \\
\text { circum-ference }\end{array}$ & 0.768 & 0.518 & 0.375 & 0.322 & 0.363 & 0.396 & 0.445 & 0.457 & 0.447 & 0.479 & 0.329 & 0.594 \\
\hline$P$ & & $<0.001$ & $<0.001$ & $<0.001$ & 0.001 & $<0.001$ & $<0.001$ & $<0.001$ & $<0.001$ & $<0.001$ & $<0.001$ & 0.001 & $<0.001$ \\
\hline$N$ & 77 & & 107 & 106 & 107 & 103 & 107 & 107 & 106 & 107 & 107 & 100 & 101 \\
\hline$\rho$ & 0.618 & Bitragion & 0.537 & 0.344 & 0.357 & 0.203 & 0.249 & 0.279 & 0.335 & 0.301 & 0.379 & 0.260 & 0.512 \\
\hline$P$ & $<0.001$ & head arc & $<0.001$ & $<0.001$ & $<0.001$ & 0.039 & 0.010 & 0.004 & $<0.001$ & 0.002 & $<0.001$ & 0.009 & $<0.001$ \\
\hline$N$ & 77 & 77 & \multirow{3}{*}{$\begin{array}{l}\text { Sagittal head } \\
\text { arc }\end{array}$} & 106 & 107 & 103 & 107 & 107 & 106 & 107 & 107 & 100 & 101 \\
\hline$\rho$ & 0.582 & 0.480 & & 0.092 & 0.270 & -0.032 & -0.027 & 0.155 & 0.195 & 0.101 & 0.094 & 0.148 & 0.303 \\
\hline$P$ & $<0.001$ & $<0.001$ & & 0.346 & 0.005 & 0.746 & 0.782 & 0.111 & 0.045 & 0.303 & 0.334 & 0.142 & 0.002 \\
\hline N & 75 & 75 & 75 & \multirow{3}{*}{$\begin{array}{c}\text { Mandibular } \\
\text { arc }\end{array}$} & 106 & 103 & 106 & 106 & 105 & 106 & 106 & 99 & 100 \\
\hline$\rho$ & 0.444 & 0.413 & 0.207 & & 0.402 & 0.361 & 0.509 & 0.591 & 0.543 & 0.513 & 0.559 & 0.267 & 0.491 \\
\hline$P$ & $<0.001$ & $<0.001$ & 0.074 & & $<0.001$ & $<0.001$ & $<0.001$ & $<0.001$ & $<0.001$ & $<0.001$ & $<0.001$ & 0.008 & $<0.001$ \\
\hline N & 77 & 77 & 77 & 75 & \multirow{3}{*}{$\begin{array}{l}\text { Lip-chin } \\
\text { distance }\end{array}$} & 103 & 107 & 107 & 106 & 107 & 107 & 100 & 101 \\
\hline$\rho$ & 0.388 & 0.222 & -0.110 & 0.364 & & 0.278 & 0.333 & 0.327 & 0.291 & 0.255 & 0.223 & 0.295 & 0.272 \\
\hline$P$ & $<0.001$ & 0.052 & 0.340 & 0.001 & & 0.005 & $<0.001$ & 0.001 & 0.002 & 0.008 & 0.021 & 0.003 & 0.006 \\
\hline$N$ & 73 & 73 & 73 & 71 & 73 & \multirow{3}{*}{$\begin{array}{c}\text { Wrist } \\
\text { circum-ference }\end{array}$} & 103 & 103 & 102 & 103 & 103 & 96 & 97 \\
\hline$\rho$ & 0.421 & 0.271 & 0.292 & 0.482 & 0.287 & & 0.514 & 0.552 & 0.515 & 0.517 & 0.579 & 0.220 & 0.361 \\
\hline$P$ & $<0.001$ & 0.020 & 0.012 & $<0.001$ & 0.014 & & $<0.001$ & $<0.001$ & $<0.001$ & $<0.001$ & $<0.001$ & 0.032 & $<0.001$ \\
\hline$N$ & 75 & 75 & 75 & 73 & 75 & 71 & \multirow{3}{*}{$\begin{array}{l}\text { Waist } \\
\text { circum-ference }\end{array}$} & 107 & 106 & 107 & 107 & 100 & 101 \\
\hline$\rho$ & 0.386 & 0.282 & 0.196 & 0.484 & 0.256 & 0.632 & & 0.761 & 0.671 & 0.724 & 0.579 & 0.201 & 0.467 \\
\hline$P$ & 0.001 & 0.014 & 0.092 & $<0.001$ & 0.027 & $<0.001$ & & $<0.001$ & $<0.001$ & $<0.001$ & $<0.001$ & 0.045 & $<0.001$ \\
\hline$N$ & 75 & 75 & 75 & 73 & 75 & 71 & 75 & \multirow{3}{*}{$\begin{array}{l}\text { Hip } \\
\text { circum-ference }\end{array}$} & 106 & 107 & 107 & 100 & 101 \\
\hline$\rho$ & 0.506 & 0.323 & 0.219 & 0.537 & 0.290 & 0.768 & 0.889 & & 0.827 & 0.762 & 0.697 & 0.242 & 0.527 \\
\hline$P$ & $<0.001$ & 0.005 & 0.059 & $<0.001$ & 0.012 & $<0.001$ & $<0.001$ & & $<0.001$ & $<0.001$ & $<0.001$ & 0.015 & $<0.001$ \\
\hline$N$ & 77 & 77 & 77 & 75 & 77 & 73 & 75 & 75 & \multirow{3}{*}{$\begin{array}{l}\text { Thigh } \\
\text { circum-ference }\end{array}$} & 106 & 106 & 99 & 100 \\
\hline$\rho$ & 0.271 & 0.077 & 0.118 & 0.384 & 0.204 & 0.653 & 0.653 & 0.739 & & 0.785 & 0.673 & 0.289 & 0.558 \\
\hline$P$ & 0.017 & 0.508 & 0.308 & 0.001 & 0.075 & $<0.001$ & $<0.001$ & $<0.001$ & & $<0.001$ & $<0.001$ & 0.004 & $<0.001$ \\
\hline$N$ & 77 & 77 & 77 & 75 & 77 & 73 & 75 & 75 & 77 & \multirow{3}{*}{$\begin{array}{c}\text { Calf } \\
\text { circum-ference }\end{array}$} & 107 & 100 & 101 \\
\hline$\rho$ & 0.309 & 0.195 & 0.253 & 0.455 & 0.095 & 0.701 & 0.611 & 0.752 & 0.735 & & 0.717 & 0.237 & 0.620 \\
\hline$P$ & 0.006 & 0.090 & 0.026 & $<0.001$ & 0.411 & $<0.001$ & $<0.001$ & $<0.001$ & $<0.001$ & & $<0.001$ & $\begin{array}{l}0.018 \\
0.018\end{array}$ & $<0.001$ \\
\hline$N$ & 77 & 77 & 77 & 75 & 77 & 73 & 75 & 75 & 77 & 77 & \multirow{3}{*}{$\begin{array}{l}\text { Ankle } \\
\text { circum-ference }\end{array}$} & 100 & 101 \\
\hline$\rho$ & 0.229 & 0.168 & 0.073 & 0.435 & 0.116 & 0.699 & 0.548 & 0.664 & 0.557 & 0.725 & & 0.445 & 0.615 \\
\hline$P$ & 0.045 & $\begin{array}{l}0.100 \\
0.145\end{array}$ & 0.530 & $<0.001$ & $\begin{array}{l}0.315 \\
0.315\end{array}$ & $<0.001$ & $<0.001$ & $<0.001$ & $<0.001$ & $<0.001$ & & $<0.001$ & $<0.001$ \\
\hline$N$ & 74 & 74 & 74 & 72 & 74 & 70 & 73 & 73 & 74 & 74 & 74 & \multirow{3}{*}{ Foot length } & 100 \\
\hline$\rho$ & 0.173 & 0.149 & 0.137 & 0.122 & 0.098 & 0.362 & 0.218 & 0.292 & 0.114 & 0.363 & 0.464 & & 0.453 \\
\hline$P$ & 0.140 & 0.206 & 0.246 & 0.306 & 0.408 & 0.002 & 0.064 & 0.012 & 0.332 & 0.001 & $<0.001$ & & $<0.001$ \\
\hline$N$ & 75 & 75 & 75 & 73 & 75 & 71 & 74 & 74 & 75 & 75 & 75 & 74 & \multirow{3}{*}{$\begin{array}{c}\text { Foot } \\
\text { breadth }\end{array}$} \\
\hline$\rho$ & 0.348 & 0.404 & 0.267 & 0.392 & 0.138 & 0.624 & 0.404 & 0.477 & 0.344 & 0.605 & 0.601 & 0.463 & \\
\hline$P$ & 0.002 & $<0.001$ & 0.021 & 0.001 & 0.239 & $<0.001$ & $<0.001$ & $<0.001$ & 0.002 & $<0.001$ & $<0.001$ & $<0.001$ & \\
\hline
\end{tabular}

Spearman correlations. $P<0.05$ in bold. 
Table A8. Inter-correlations between male body measurements residualized on height (alcohol-dependent in-patients bottom left, healthy control subjects top right).

\begin{tabular}{|c|c|c|c|c|c|c|c|c|c|c|c|c|c|}
\hline $\bar{N}$ & & 133 & 133 & 133 & 132 & 128 & 133 & 133 & 133 & 132 & 131 & 105 & 107 \\
\hline$\rho$ & Head & 0.459 & 0.384 & 0.125 & 0.246 & 0.390 & 0.275 & 0.232 & 0.228 & 0.333 & 0.379 & 0.336 & 0.226 \\
\hline$P$ & & $<0.001$ & $<0.001$ & 0.153 & 0.005 & $<0.001$ & 0.001 & 0.007 & 0.008 & $<0.001$ & $<0.001$ & $<0.001$ & 0.019 \\
\hline$N$ & 107 & & 133 & 133 & 132 & 128 & 133 & 133 & 133 & 132 & 131 & 105 & 107 \\
\hline$\rho$ & 0.309 & Bitragion & 0.323 & -0.040 & 0.097 & 0.142 & 0.063 & 0.047 & 0.048 & 0.041 & 0.176 & 0.227 & 0.101 \\
\hline$P$ & 0.001 & head arc & $<0.001$ & 0.651 & 0.269 & 0.109 & 0.470 & 0.591 & 0.584 & 0.642 & 0.044 & 0.020 & 0.300 \\
\hline$N$ & 107 & 107 & \multirow{3}{*}{$\begin{array}{l}\text { Sagittal } \\
\text { head arc }\end{array}$} & 133 & 132 & 128 & 133 & 133 & 133 & 132 & 131 & 105 & 107 \\
\hline$\rho$ & 0.239 & 0.251 & & -0.015 & 0.136 & 0.013 & -0.073 & -0.023 & 0.069 & 0.074 & 0.130 & 0.035 & -0.041 \\
\hline$P$ & 0.013 & 0.009 & & 0.864 & 0.120 & 0.881 & 0.403 & 0.794 & 0.432 & 0.401 & 0.138 & 0.725 & 0.673 \\
\hline$N$ & 106 & 106 & 106 & \multirow{3}{*}{$\begin{array}{c}\text { Mandibular } \\
\text { arc }\end{array}$} & 132 & 128 & 133 & 133 & 133 & 132 & 131 & 105 & 107 \\
\hline$\rho$ & 0.307 & 0.086 & -0.139 & & 0.320 & 0.355 & 0.245 & 0.156 & 0.096 & 0.291 & 0.305 & 0.202 & 0.096 \\
\hline$P$ & 0.001 & 0.383 & 0.156 & & $<0.001$ & $<0.001$ & 0.004 & 0.073 & 0.273 & 0.001 & $<0.001$ & 0.039 & 0.326 \\
\hline$N$ & 106 & 106 & 106 & 105 & \multirow{3}{*}{$\begin{array}{l}\text { Lip-chin } \\
\text { distance }\end{array}$} & 127 & 132 & 132 & 132 & 131 & 130 & 104 & 106 \\
\hline$\rho$ & 0.355 & 0.089 & -0.278 & 0.420 & & 0.209 & 0.158 & 0.136 & 0.204 & 0.248 & 0.181 & 0.205 & 0.104 \\
\hline$P$ & $<0.001$ & 0.365 & 0.004 & $<0.001$ & & 0.018 & 0.070 & 0.121 & 0.019 & 0.004 & 0.039 & 0.037 & 0.291 \\
\hline$N$ & 102 & 102 & 102 & 101 & 101 & & 128 & 128 & 128 & 127 & 126 & 101 & 103 \\
\hline$\rho$ & 0.404 & 0.112 & $\begin{array}{l}10.105 \\
-0.105\end{array}$ & 0.434 & 0.384 & Wrist & 0.578 & 0.509 & 0.418 & 0.522 & 0.533 & 0.270 & 0.372 \\
\hline$P$ & $<0.001$ & 0.262 & 0.295 & $<0.001$ & $<0.001$ & circum-ference & $<0.001$ & $<0.001$ & $<0.001$ & $<0.001$ & $<0.001$ & 0.006 & $<0.001$ \\
\hline$N$ & 106 & 106 & 106 & 105 & 105 & 102 & \multirow{3}{*}{$\begin{array}{l}\text { Waist } \\
\text { circum-ference }\end{array}$} & 133 & 133 & 132 & 131 & 105 & 107 \\
\hline$\rho$ & 0.401 & 0.152 & -0.141 & 0.398 & 0.273 & 0.549 & & 0.732 & 0.551 & 0.572 & 0.399 & 0.179 & 0.298 \\
\hline$P$ & $<0.001$ & 0.120 & 0.149 & $<0.001$ & 0.005 & $<0.001$ & & $<0.001$ & $<0.001$ & $<0.001$ & $<0.001$ & 0.068 & 0.002 \\
\hline$N$ & 106 & 106 & 106 & 105 & 105 & 102 & 106 & \multirow{3}{*}{$\begin{array}{l}\text { Hip } \\
\text { circum-ference }\end{array}$} & 133 & 132 & 131 & 105 & 107 \\
\hline$\rho$ & 0.350 & 0.151 & -0.096 & 0.350 & 0.212 & 0.494 & 0.796 & & 0.763 & 0.612 & 0.397 & 0.071 & 0.233 \\
\hline$P$ & $<0.001$ & 0.122 & 0.326 & $<0.001$ & 0.030 & $<0.001$ & $<0.001$ & & $<0.001$ & $<0.001$ & $<0.001$ & 0.474 & 0.016 \\
\hline$N$ & 106 & 106 & 106 & 105 & 105 & 102 & 106 & 106 & \multirow{3}{*}{$\begin{array}{l}\text { Thigh } \\
\text { circum-ference }\end{array}$} & 132 & 131 & 105 & 107 \\
\hline$\rho$ & 0.381 & -0.042 & -0.089 & 0.370 & 0.264 & 0.522 & 0.715 & 0.709 & & $\begin{array}{l}152 \\
0.666\end{array}$ & $\begin{array}{l}151 \\
0.362\end{array}$ & -0.122 & 0.152 \\
\hline$P$ & $<0.001$ & 0.671 & 0.365 & $<0.001$ & 0.007 & $<0.001$ & $<0.001$ & $<0.001$ & & $<0.001$ & $<0.001$ & 0.216 & 0.117 \\
\hline$N$ & 106 & 106 & 106 & 105 & 105 & 102 & 106 & 106 & 106 & & 131 & 104 & 106 \\
\hline$\rho$ & 0.282 & 0.066 & $\begin{array}{l}100 \\
-0.010\end{array}$ & 0.298 & 0.160 & 0.573 & 0.609 & 0.545 & 0.689 & & 0.447 & $\begin{array}{l}104 \\
0.042\end{array}$ & 0.356 \\
\hline$P$ & 0.003 & 0.502 & 0.920 & 0.002 & 0.104 & $<0.001$ & $<0.001$ & $<0.001$ & $\begin{array}{l}0.009 \\
<0.001\end{array}$ & circum-ference & $\begin{array}{l}0.44 / \\
<0.001\end{array}$ & $\begin{array}{l}0.042 \\
0.669\end{array}$ & $\begin{array}{l}0.356 \\
<0.001\end{array}$ \\
\hline$N$ & 106 & 106 & 106 & 105 & 105 & 102 & 106 & 106 & 106 & 106 & & 103 & 105 \\
\hline$\rho$ & 0.396 & 0.168 & -0.171 & 0.398 & 0.435 & 0.562 & 0.540 & 0.501 & 0.519 & 0.616 & Ankle & 0.382 & 0.495 \\
\hline$P$ & $<0.001$ & 0.085 & 0.080 & $<0.001$ & $<0.001$ & $<0.001$ & $<0.001$ & $<0.001$ & $<0.001$ & $<0.001$ & circum-ference & $<0.001$ & $<0.001$ \\
\hline$N$ & 99 & 99 & 99 & 98 & 98 & 95 & 98 & 98 & 98 & 98 & 98 & & 105 \\
\hline$\rho$ & 0.190 & 0.082 & 0.018 & 0.306 & 0.370 & 0.370 & 0.303 & 0.284 & 0.260 & 0.293 & 0.505 & Foot length & 0.356 \\
\hline$P$ & 0.060 & 0.419 & 0.856 & 0.002 & $<0.001$ & $<0.001$ & 0.002 & 0.005 & 0.010 & 0.003 & $<0.001$ & & $<0.001$ \\
\hline$N$ & 100 & 100 & 100 & 99 & 99 & 95 & 99 & 99 & 99 & 99 & 99 & 99 & \\
\hline$\rho$ & 0.304 & 0.166 & -0.085 & 0.221 & 0.257 & 0.519 & 0.367 & 0.341 & 0.317 & 0.448 & 0.584 & 0.428 & Foot \\
\hline$P$ & 0.002 & 0.099 & 0.400 & 0.028 & 0.010 & $<0.001$ & $<0.001$ & 0.001 & 0.001 & $<0.001$ & $<0.001$ & $<0.001$ & breadth \\
\hline
\end{tabular}

Spearman correlations. $P<0.05$ in bold. 
Table A9. Inter-correlations between female body measurements residualized on height (alcohol-dependent in-patients bottom left, healthy control subjects top right).

\begin{tabular}{|c|c|c|c|c|c|c|c|c|c|c|c|c|c|}
\hline$N$ & & 107 & 107 & 106 & 107 & 103 & 107 & 107 & 106 & 107 & 107 & 100 & 101 \\
\hline$\rho$ & Head & 0.652 & 0.372 & 0.219 & 0.265 & 0.231 & 0.267 & 0.290 & 0.301 & 0.245 & 0.296 & 0.210 & 0.354 \\
\hline$P$ & circum-ference & $<0.001$ & $<0.001$ & 0.024 & 0.006 & 0.019 & 0.005 & 0.002 & 0.002 & 0.011 & 0.002 & 0.036 & $<0.001$ \\
\hline N & 77 & \multirow{3}{*}{$\begin{array}{l}\text { Bitragion } \\
\text { head arc }\end{array}$} & 107 & 106 & 107 & 103 & 107 & 107 & 106 & 107 & 107 & 100 & 101 \\
\hline$\rho$ & 0.380 & & 0.388 & 0.205 & 0.283 & 0.060 & 0.095 & 0.023 & 0.102 & 0.064 & 0.198 & 0.165 & 0.324 \\
\hline$P$ & 0.001 & & $<0.001$ & 0.035 & 0.003 & 0.549 & 0.332 & 0.811 & 0.296 & 0.515 & 0.041 & 0.101 & 0.001 \\
\hline N & 77 & 77 & \multirow{3}{*}{$\begin{array}{l}\text { Sagittal } \\
\text { head arc }\end{array}$} & 106 & 107 & 103 & 107 & 107 & 106 & 107 & 107 & 100 & 101 \\
\hline$\rho$ & 0.351 & 0.303 & & -0.056 & 0.187 & -0.170 & -0.148 & 0.000 & 0.067 & -0.057 & -0.075 & 0.061 & 0.111 \\
\hline$P$ & 0.002 & 0.007 & & 0.567 & 0.054 & 0.086 & 0.128 & 0.996 & 0.493 & 0.563 & 0.441 & 0.548 & 0.269 \\
\hline N & 75 & 75 & 75 & \multirow{3}{*}{$\begin{array}{l}\text { Mandibular } \\
\text { arc }\end{array}$} & 106 & 103 & 106 & 106 & 105 & 106 & 106 & 99 & 100 \\
\hline$\rho$ & 0.294 & 0.268 & 0.049 & & 0.350 & 0.334 & 0.478 & 0.469 & 0.443 & 0.438 & 0.497 & 0.236 & 0.404 \\
\hline$P$ & 0.011 & 0.020 & 0.676 & & $<0.001$ & 0.001 & $<0.001$ & $<0.001$ & $<0.001$ & $<0.001$ & $<0.001$ & 0.019 & $<0.001$ \\
\hline N & 77 & $\begin{array}{l}0.020 \\
77\end{array}$ & 77 & 75 & \multirow{2}{*}{$\begin{array}{l}\text { Lip-chin } \\
\text { distance }\end{array}$} & 103 & 107 & 107 & 106 & 107 & 107 & 100 & 101 \\
\hline$P$ & 0.014 & 0.643 & 0.003 & 0.011 & & 0.009 & 0.002 & 0.036 & 0.028 & 0.080 & 0.137 & 0.005 & 0.057 \\
\hline N & 73 & 73 & 73 & 71 & 73 & \multirow{3}{*}{$\begin{array}{l}\text { Wrist } \\
\text { circum-ference }\end{array}$} & 103 & 103 & 102 & 103 & 103 & 96 & 97 \\
\hline$\rho$ & 0.291 & 0.120 & 0.180 & 0.429 & 0.258 & & 0.499 & 0.458 & 0.401 & 0.452 & 0.521 & 0.217 & 0.324 \\
\hline$P$ & 0.012 & 0.310 & 0.128 & $<0.001$ & 0.028 & & $<0.001$ & $<0.001$ & $<0.001$ & $<0.001$ & $<0.001$ & 0.033 & 0.001 \\
\hline N & 75 & 75 & 75 & 73 & 75 & 71 & \multirow{3}{*}{$\begin{array}{l}\text { Waist } \\
\text { circum-ference }\end{array}$} & 107 & 106 & 107 & 107 & 100 & 101 \\
\hline$\rho$ & 0.304 & 0.163 & 0.095 & 0.417 & 0.213 & 0.620 & & 0.695 & 0.622 & 0.684 & 0.507 & 0.201 & 0.413 \\
\hline$P$ & 0.008 & 0.162 & 0.418 & $<0.001$ & 0.066 & $<0.001$ & & $<0.001$ & $<0.001$ & $<0.001$ & $<0.001$ & 0.045 & $<0.001$ \\
\hline N & 75 & 75 & 75 & 73 & 75 & 71 & 75 & \multirow{3}{*}{$\begin{array}{c}\text { Hip } \\
\text { circum-ference }\end{array}$} & 106 & 107 & 107 & 100 & 101 \\
\hline$\rho$ & 0.382 & 0.207 & 0.035 & 0.446 & 0.210 & 0.671 & 0.863 & & 0.838 & 0.722 & 0.589 & 0.135 & 0.354 \\
\hline$P$ & 0.001 & 0.074 & 0.767 & $<0.001$ & 0.071 & $<0.001$ & $<0.001$ & & $<0.001$ & $<0.001$ & $<0.001$ & 0.181 & $<0.001$ \\
\hline N & 77 & 77 & 77 & 75 & 77 & 73 & 75 & 75 & \multirow{3}{*}{$\begin{array}{l}\text { Thigh } \\
\text { circum-ference }\end{array}$} & 106 & 106 & $\begin{array}{l}0.101 \\
99\end{array}$ & 100 \\
\hline$\rho$ & 0.207 & -0.023 & 0.030 & 0.296 & 0.099 & 0.541 & 0.590 & 0.727 & & 0.746 & 0.562 & 0.193 & 0.410 \\
\hline$P$ & 0.071 & 0.844 & 0.796 & 0.010 & 0.392 & $<0.001$ & $<0.001$ & $<0.001$ & & $<0.001$ & $<0.001$ & 0.055 & $<0.001$ \\
\hline$P$ & 0.062 & 0.342 & 0.101 & $<0.001$ & 0.784 & $<0.001$ & $<0.001$ & $<0.001$ & $<0.001$ & $\begin{array}{c}\text { Calf } \\
\text { circum-ference }\end{array}$ & \multirow{4}{*}{$\begin{array}{c}\text { Ankle } \\
\text { circum-ference }\end{array}$} & 0.083 & $<0.001$ \\
\hline N & 77 & 77 & 77 & 75 & 77 & 73 & 75 & 75 & 77 & 77 & & 100 & 101 \\
\hline $\begin{array}{c}N \\
\rho\end{array}$ & 0.157 & 0.115 & 0.058 & 0.388 & 0.032 & 0.639 & 0.511 & 0.634 & 0.542 & 0.725 & & 0.432 & $\begin{array}{l}101 \\
0.533\end{array}$ \\
\hline$P$ & 0.172 & 0.318 & 0.615 & 0.001 & 0.783 & $<0.001$ & $<0.001$ & $<0.001$ & $<0.001$ & $<0.001$ & & $<0.001$ & $<0.001$ \\
\hline N & 74 & 74 & 74 & 72 & 74 & 70 & 73 & 73 & 74 & 74 & 74 & \multirow{3}{*}{ Foot length } & 100 \\
\hline$\rho$ & 0.212 & 0.180 & 0.176 & 0.130 & 0.112 & 0.403 & 0.247 & 0.294 & 0.098 & 0.369 & 0.474 & & 0.453 \\
\hline$P$ & 0.070 & 0.125 & 0.134 & 0.275 & 0.342 & 0.001 & 0.035 & 0.012 & 0.409 & 0.001 & $<0.001$ & & $<0.001$ \\
\hline N & 75 & 75 & $\begin{array}{l}7.104 \\
75\end{array}$ & 73 & 75 & 71 & $\begin{array}{l}74 \\
74\end{array}$ & 74 & 75 & 75 & 75 & 74 & \\
\hline$\rho$ & 0.153 & 0.240 & 0.132 & 0.331 & 0.088 & 0.585 & 0.371 & 0.378 & 0.263 & 0.559 & 0.569 & 0.506 & Foot breadth \\
\hline$P$ & 0.190 & 0.038 & 0.258 & 0.004 & 0.452 & $<0.001$ & 0.001 & 0.001 & 0.022 & $<0.001$ & $<0.001$ & $<0.001$ & \\
\hline
\end{tabular}

Spearman correlations. $P<0.05$ in bold. 


\section{References}

1. World Health Organization. Global Status Report on Alcohol and Health 2018. 2018. Available online: https://www.who.int/substance_abuse/publications/global_alcohol_report/gsr_2018/en/ (accessed on 1 January 2019).

2. Weinland, C.; Mühle, C.; Kornhuber, J.; Lenz, B. Crossed eye/hand laterality and left-eyedness predict a positive 24-month outcome in alcohol-dependent patients. Alcohol. Clin. Exp. Res. 2019, 43, 1308-1317. [CrossRef] [PubMed]

3. Urban, N.B.L.; Kegeles, L.S.; Slifstein, M.; Xu, X.; Martinez, D.; Sakr, E.; Castillo, F.; Moadel, T.; O'Malley, S.S.; Krystal, J.H.; et al. Sex differences in striatal dopamine release in young adults after oral alcohol challenge: A positron emission tomography imaging study with [ $\left.{ }^{11} \mathrm{C}\right]$ raclopride. Biol. Psychiatry 2010, 68, 689-696. [CrossRef] [PubMed]

4. Treutlein, J.; Cichon, S.; Ridinger, M.; Wodarz, N.; Soyka, M.; Zill, P.; Maier, W.; Moessner, R.; Gaebel, W.; Dahmen, N.; et al. Genome-wide association study of alcohol dependence. Arch. Gen. Psychiatry 2009, 66, 773-784. [CrossRef] [PubMed]

5. Mühle, C.; Barry, B.; Weinland, C.; Kornhuber, J.; Lenz, B. Estrogen receptor 1 gene variants and estradiol activities in alcohol dependence. Prog. Neuropsychopharmacol. Biol. Psychiatry 2019, 92, 301-307. [CrossRef]

6. Lenz, B.; Jacob, C.; Frieling, H.; Jacobi, A.; Hillemacher, T.; Muschler, M.; Watson, K.; Kornhuber, J.; Bleich, S. Polymorphism of the long polyglutamine tract in the human androgen receptor influences craving of men in alcohol withdrawal. Psychoneuroendocrinology 2009, 34, 968-971. [CrossRef]

7. Lenz, B.; Frieling, H.; Jacob, C.; Heberlein, A.; Kornhuber, J.; Bleich, S.; Hillemacher, T. The modulating effect of the androgen receptor on craving in alcohol withdrawal of men is partially mediated by leptin. Pharmacogenomics J. 2010, 10, 226-231. [CrossRef]

8. Lenz, B.; Heberlein, A.; Bayerlein, K.; Frieling, H.; Kornhuber, J.; Bleich, S.; Hillemacher, T. The TTTAn aromatase (CYP19A1) polymorphism is associated with compulsive craving of male patients during alcohol withdrawal. Psychoneuroendocrinology 2011, 36, 1261-1264. [CrossRef]

9. Milivojevic, V.; Kranzler, H.R.; Gelernter, J.; Burian, L.; Covault, J. Variation in genes encoding the neuroactive steroid synthetic enzymes $5 \alpha$-reductase type 1 and $3 \alpha$-reductase type 2 is associated with alcohol dependence. Alcohol. Clin. Exp. Res. 2011, 35, 946-952. [CrossRef]

10. Lenz, B.; Schöpp, E.; Müller, C.P.; Bleich, S.; Hillemacher, T.; Kornhuber, J. Association of V89L SRD5A2 polymorphism with craving and serum leptin levels in male alcohol addicts. Psychopharmacology 2012, 224, 421-429. [CrossRef]

11. Huber, S.E.; Lenz, B.; Kornhuber, J.; Müller, C.P. Prenatal androgen-receptor activity has organizational morphological effects in mice. PLoS ONE 2017, 12, e0188752. [CrossRef]

12. Huber, S.E.; Zoicas, I.; Reichel, M.; Mühle, C.; Büttner, C.; Ekici, A.B.; Eulenburg, V.; Lenz, B.; Kornhuber, J.; Müller, C.P. Prenatal androgen receptor activation determines adult alcohol and water drinking in a sex-specific way. Addict. Biol. 2018, 23, 904-920. [CrossRef] [PubMed]

13. Schulz, K.M.; Sisk, C.L. The organizing actions of adolescent gonadal steroid hormones on brain and behavioral development. Neurosci. Biobehav. Rev. 2016, 70, 148-158. [CrossRef] [PubMed]

14. Manning, J.; Kilduff, L.; Cook, C.; Crewther, B.; Fink, B. Digit ratio (2D:4D): A biomarker for prenatal sex steroids and adult sex steroids in challenge situations. Front. Endocrinol. 2014, 5, 9. [CrossRef] [PubMed]

15. Zheng, Z.; Cohn, M.J. Developmental basis of sexually dimorphic digit ratios. Proc. Natl. Acad. Sci. USA 2011, 108, 16289-16294. [CrossRef] [PubMed]

16. Berenbaum, S.A.; Bryk, K.K.; Nowak, N.; Quigley, C.A.; Moffat, S. Fingers as a marker of prenatal androgen exposure. Endocrinology 2009, 150, 5119-5124. [CrossRef]

17. Del Giudice, M.; Barrett, E.S.; Belsky, J.; Hartman, S.; Martel, M.M.; Sangenstedt, S.; Kuzawa, C.W. Individual differences in developmental plasticity: A role for early androgens? Psychoneuroendocrinology 2018, 90, 165-173. [CrossRef]

18. Eichler, A.; Heinrich, H.; Moll, G.H.; Beckmann, M.W.; Goecke, T.W.; Fasching, P.A.; Muschler, M.R.; Bouna-Pyrrou, P.; Lenz, B.; Kornhuber, J. Digit ratio (2D:4D) and behavioral symptoms in primary-school aged boys. Early Hum. Dev. 2018, 119, 1-7. [CrossRef] 
19. Kornhuber, J.; Zenses, E.M.; Lenz, B.; Stoessel, C.; Bouna-Pyrrou, P.; Rehbein, F.; Kliem, S.; Mößle, T. Low 2D:4D values are associated with video game addiction. PLoS ONE 2013, 8, e79539. [CrossRef]

20. Canan, F.; Karaca, S.; Düzgün, M.; Erdem, A.M.; Karaçaylı, E.; Topan, N.B.; Lee, S.K.; Zhai, Z.W.; Kuloğlu, M.; Potenza, M.N. The relationship between second-to-fourth digit (2D:4D) ratios and problematic and pathological Internet use among Turkish university students. J. Behav. Addict. 2017, 6, 30-41. [CrossRef]

21. Lenz, B.; Röther, M.; Bouna-Pyrrou, P.; Mühle, C.; Tektas, O.Y.; Kornhuber, J. The androgen model of suicide completion. Prog. Neurobiol. 2019, 172, 84-103. [CrossRef]

22. Lenz, B.; Thiem, D.; Bouna-Pyrrou, P.; Mühle, C.; Stoessel, C.; Betz, P.; Kornhuber, J. Low digit ratio (2D:4D) in male suicide victims. J. Neural Transm. 2016, 123, 1499-1503. [CrossRef] [PubMed]

23. Lenz, B.; Kornhuber, J. Cross-national gender variations of digit ratio (2D:4D) correlate with life expectancy, suicide rate, and other causes of death. J. Neural Transm. 2018, 125, 239-246. [CrossRef] [PubMed]

24. Lenz, B.; Bouna-Pyrrou, P.; Mühle, C.; Kornhuber, J. Low digit ratio (2D:4D) and late pubertal onset indicate prenatal hyperandrogenziation in alcohol binge drinking. Prog. Neuropsychopharmacol. Biol. Psychiatry 2018, 86, 370-378. [CrossRef] [PubMed]

25. Lenz, B.; Mühle, C.; Cohort Study on Substance Use Risk Factors; Kornhuber, J. Lower digit ratio (2D:4D) in alcohol dependence: Confirmation and exploratory analysis in a population-based study of young men. Addict. Biol. 2019, e12815. [CrossRef] [PubMed]

26. Manning, J.T.; Fink, B. Digit ratio, nicotine and alcohol intake and national rates of smoking and alcohol consumption. Personal. Individ. Differ. 2011, 50, 344-348. [CrossRef]

27. Canan, F.; Tegin, C.; Gecici, O. The second to fourth digit (2D:4D) ratios, smoking, and problem drinking in a young adult university student sample. Neurol. Psychiatry Brain Res. 2019, 32, 63-67. [CrossRef]

28. Kornhuber, J.; Erhard, G.; Lenz, B.; Kraus, T.; Sperling, W.; Bayerlein, K.; Biermann, T.; Stoessel, C. Low digit ratio 2D:4D in alcohol dependent patients. PLoS ONE 2011, 6, e19332. [CrossRef]

29. Lenz, B.; Mühle, C.; Braun, B.; Weinland, C.; Bouna-Pyrrou, P.; Behrens, J.; Kubis, S.; Mikolaiczik, K.; Muschler, M.-R.; Saigali, S.; et al. Prenatal and adult androgen activities in alcohol dependence. Acta Psychiatr. Scand. 2017, 136, 96-107. [CrossRef]

30. Han, C.; Bae, H.; Lee, Y.-S.; Won, S.-D.; Kim, D.J. The ratio of 2nd to 4th digit length in Korean alcohol-dependent patients. Clin. Psychopharmacol. Neurosci. 2016, 14, 148-152. [CrossRef]

31. Siegmann, E.M.; Bouna-Pyrrou, P.; Lenz, B.; Kornhuber, J. Digit ratio (2D:4D) in relation to substance and computer use: A meta-analysis. J. Neural Transm. 2019, 126, 623-636. [CrossRef]

32. Lenz, B.; Müller, C.P.; Stoessel, C.; Sperling, W.; Biermann, T.; Hillemacher, T.; Bleich, S.; Kornhuber, J. Sex hormone activity in alcohol addiction: Integrating organizational and activational effects. Prog. Neurobiol. 2012, 96, 136-163. [CrossRef] [PubMed]

33. Gegenhuber, B.; Weinland, C.; Kornhuber, J.; Mühle, C.; Lenz, B. OPRM1 A118G and serum $\beta$-endorphin interact with sex and digit ratio (2D:4D) to influence risk and course of alcohol dependence. Eur. Neuropsychopharmacol. 2018, 28, 1418-1428. [CrossRef] [PubMed]

34. Cohen-Bendahan, C.C.C.; van de Beek, C.; Berenbaum, S.A. Prenatal sex hormone effects on child and adult sex-typed behavior: Methods and findings. Neurosci. Biobehav. Rev. 2005, 29, 353-384. [CrossRef] [PubMed]

35. Tapp, A.L.; Maybery, M.T.; Whitehouse, A.J.O. Evaluating the twin testosterone transfer hypothesis: A review of the empirical evidence. Horm. Behav. 2011, 60, 713-722. [CrossRef]

36. Lenz, B.; Müller, C.P.; Kornhuber, J. Alcohol dependence in same-sex and opposite-sex twins. J. Neural Transm. 2012, 119, 1561-1564. [CrossRef]

37. Ellingson, J.M.; Slutske, W.S.; Richmond-Rakerd, L.S.; Martin, N.G. Investigating the influence of prenatal androgen exposure and sibling effects on alcohol use and alcohol use disorder in females from opposite-sex twin pairs. Alcohol. Clin. Exp. Res. 2013, 37, 868-876. [CrossRef]

38. Malas, M.A.; Dogan, S.; Evcil, E.H.; Desdicioglu, K. Fetal development of the hand, digits and digit ratio (2D:4D). Early Hum. Dev. 2006, 82, 469-475. [CrossRef]

39. Galis, F.; Ten Broek, C.M.A.; Van Dongen, S.; Wijnaendts, L.C.D. Sexual dimorphism in the prenatal digit ratio (2D:4D). Arch. Sex. Behav. 2010, 39, 57-62. [CrossRef] 
40. Lenz, B.; Eichler, A.; Schwenke, E.; Buchholz, V.N.; Hartwig, C.; Moll, G.H.; Reich, K.; Mühle, C.; Volz, B.; Titzmann, A.; et al. Mindfulness-based stress reduction in pregnancy: An app-based programme to improve the health of mothers and children (MINDFUL/PMI Study). Geburtshilfe Frauenheilkd. 2018, 78, 1282-1291. [CrossRef]

41. Weston, E.M.; Friday, A.E.; Liò, P. Biometric evidence that sexual selection has shaped the hominin face. PLoS ONE 2007, 2, e710. [CrossRef]

42. Haselhuhn, M.P.; Ormiston, M.E.; Wong, E.M. Men's facial width-to-height ratio predicts aggression: A meta-analysis. PLoS ONE 2015, 10, e0122637. [CrossRef] [PubMed]

43. Geniole, S.N.; Denson, T.F.; Dixson, B.J.; Carré, J.M.; McCormick, C.M. Evidence from meta-analyses of the facial width-to-height ratio as an evolved cue of threat. PLoS ONE 2015, 10, e0132726. [CrossRef] [PubMed]

44. World Health Organization. International Statistical Classification of Diseases and Related Health Problems, 10th Revision (ICD-10); World Health Organization: Geneva, Switzerland, 1992.

45. American Psychiatric Association. Diagnostic and Statistical Manual of Mental Disorders, 5th ed.; American Psychiatric Association: Washington, DC, USA, 2013.

46. Ewing, J.A. Detecting alcoholism. The CAGE questionnaire. JAMA 1984, 252, 1905-1907. [CrossRef] [PubMed]

47. Rumpf, H.-J.; Meyer, C.; Hapke, U.; John, U. Deutsche Version des Alcohol Use Disorders Identification Test (AUDIT-G-L). In Elektronisches Handbuch zu Erhebungsinstrumenten im Suchtbereich (EHES), Version 3.00; Glöckner-Rist, A., Rist, F., Küfner, H., Eds.; Zentrum für Umfragen, Methoden und Analysen: Mannheim, Germany, 2003.

48. Skinner, H.A. Lifetime Drinking History: Administration and Scoring Guidelines. Addiction Research Foundation. 1979. Available online: http://www.emcdda.europa.eu/html.cfm/index4163EN.html (accessed on 28 August 2012).

49. Weinland, C.; Braun, B.; Mühle, C.; Kornhuber, J.; Lenz, B. Cloninger type 2 score and Lesch typology predict hospital readmission of female and male alcohol-dependent inpatients during a 24-month follow-up. Alcohol. Clin. Exp. Res. 2017, 41, 1760-1767. [CrossRef]

50. Braun, B.; Weinland, C.; Kornhuber, J.; Lenz, B. Religiosity, guilt, altruism and forgiveness in alcohol dependence: Results of a cross-sectional and prospective cohort study. Alcohol Alcohol. 2018, 53, 426-434. [CrossRef]

51. Mühle, C.; Weinland, C.; Gulbins, E.; Lenz, B.; Kornhuber, J. Peripheral acid sphingomyelinase activity is associated with biomarkers and phenotypes of alcohol use and dependence in patients and healthy controls. Int. J. Mol. Sci. 2018, 19, 4028. [CrossRef]

52. Weinland, C.; Mühle, C.; Kornhuber, J.; Lenz, B. Body mass index and craving predict 24-month hospital readmissions of alcohol-dependent in-patients following withdrawal. Prog. Neuropsychopharmacol. Biol. Psychiatry 2019, 90, 300-307. [CrossRef]

53. Loth, S.R.; Henneberg, M. Mandibular ramus flexure: A new morphologic indicator of sexual dimorphism in the human skeleton. Am. J. Phys. Anthropol. 1996, 99, 473-485. [CrossRef]

54. Gray, J.P.; Wolfe, L.D. Height and sexual dimorphism of stature among human societies. Am. J. Phys. Anthropol. 1980, 53, 441-456. [CrossRef]

55. Wizemann, T.M.; Pardue, M.L. (Eds.) Exploring the Biological Contributions to Human Health: Does Sex Matter? National Academies Press: Washington, DC, USA, 2001.

56. Apicella, C.L.; Dreber, A.; Campbell, B.; Gray, P.B.; Hoffman, M.; Little, A.C. Testosterone and financial risk preferences. Evol. Hum. Behav. 2008, 29, 384-390. [CrossRef]

57. Burriss, R.P.; Little, A.C.; Nelson, E.C. 2D:4D and sexually dimorphic facial characteristics. Arch. Sex. Behav. 2007, 36, 377-384. [CrossRef] [PubMed]

58. Fink, B.; Neave, N.; Manning, J.T. Second to fourth digit ratio, body mass index, waist-to-hip ratio, and waist-to-chest ratio: Their relationships in heterosexual men and women. Ann. Hum. Biol. 2003, 30, 728-738. [CrossRef] [PubMed]

59. Pike, N. Using false discovery rates for multiple comparisons in ecology and evolution. Methods Ecol. Evol. 2011, 2, 278-282. [CrossRef]

60. Benjamini, Y.; Hochberg, Y. Controlling the false discovery rate: A practical and powerful approach to multiple testing. J. R. Statist. Soc. B 1995, 57, 289-300. [CrossRef] 
61. York, J.L.; Pendergast, D.E. Body composition in detoxified alcoholics. Alcohol. Clin. Exp. Res. 1990, 14, 180-183. [CrossRef]

62. York, J.L.; Welte, J.W. Gender comparisons of alcohol consumption in alcoholic and nonalcoholic populations. J. Stud. Alcohol. 1994, 55, 743-750. [CrossRef]

63. Bogl, L.H.; Jelenkovic, A.; Vuoksimaa, E.; Ahrenfeldt, L.; Pietiläinen, K.H.; Stazi, M.A.; Fagnani, C.; D'Ippolito, C.; Hur, Y.M.; Jeong, H.U.; et al. Does the sex of one's co-twin affect height and BMI in adulthood? A study of dizygotic adult twins from 31 cohorts. Biol. Sex Differ. 2017, 8, 14. [CrossRef]

64. Bay, K.; Andersson, A.M.; Skakkebaek, N.E. Estradiol levels in prepubertal boys and girls-Analytical challenges. Int. J. Androl. 2004, 27, 266-273. [CrossRef]

65. Callewaert, F.; Sinnesael, M.; Gielen, E.; Boonen, S.; Vanderschueren, D. Skeletal sexual dimorphism: relative contribution of sex steroids, GH-IGF1, and mechanical loading. J. Endocrinol. 2010, 207, 127-134. [CrossRef]

66. Callewaert, F.; Venken, K.; Kopchick, J.J.; Torcasio, A.; van Lenthe, G.H.; Boonen, S.; Vanderschueren, D. Sexual dimorphism in cortical bone size and strength but not density is determined by independent and time-specific actions of sex steroids and IGF-1: Evidence from pubertal mouse models. J. Bone Miner. Res. 2010, 25, 617-626. [CrossRef]

67. Cutler, G.B., Jr. The role of estrogen in bone growth and maturation during childhood and adolescence. J. Steroid Biochem. Mol. Biol. 1997, 61, 141-144. [CrossRef]

68. Gavaler, J.S. Alcoholic beverages as a source of estrogens. Alcohol Health Res. World 1998, 22, $220-227$. [PubMed]

69. Sarkola, T.; Eriksson, C.J.P. Testosterone increases in men after a low dose of alcohol. Alcohol. Clin. Exp. Res. 2003, 27, 682-685. [CrossRef] [PubMed]

70. Eriksson, C.J.P.; Fukunaga, T.; Lindman, R. Sex hormone response to alcohol. Nature 1994, 369, 711. [CrossRef] [PubMed]

71. Köllner, M.G.; Janson, K.T.; Bleck, K. The social biopsychology of implicit motive development. In Routledge International Handbook of Social Neuroendocrinology; Schultheiss, O.C., Mehta, P.H., Eds.; Routledge: Abingdon, UK, 2019; pp. 568-585.

72. Ge, X.; Natsuaki, M.N.; Neiderhiser, J.M.; Reiss, D. Genetic and environmental influences on pubertal timing: Results from two national sibling studies. J. Res. Adolesc. 2007, 17, 767-788. [CrossRef]

73. Popova, S.; Lange, S.; Probst, C.; Gmel, G.; Rehm, J. Estimation of national, regional, and global prevalence of alcohol use during pregnancy and fetal alcohol syndrome: A systematic review and meta-analysis. Lancet Glob. Health 2017, 5, e290-e299. [CrossRef]

74. Spohr, H.L.; Willms, J.; Steinhausen, H.C. Fetal alcohol spectrum disorders in young adulthood. J. Pediatr. 2007, 150, 175-179. [CrossRef]

75. Kraus, L.; Seitz, N.N.; Shield, K.D.; Gmel, G.; Rehm, J. Quantifying harms to others due to alcohol consumption in Germany: A register-based study. BMC Med. 2019, 17, 59. [CrossRef]

76. Zahr, N.M.; Carr, R.A.; Rohlfing, T.; Mayer, D.; Sullivan, E.V.; Colrain, I.M.; Pfefferbaum, A. Brain metabolite levels in recently sober individuals with alcohol use disorder: Relation to drinking variables and relapse. Psychiatry Res. Neuroimaging 2016, 250, 42-49. [CrossRef]

77. Gazdzinski, S.; Kornak, J.; Weiner, M.W.; Meyerhoff, D.J. Body mass index and magnetic resonance markers of brain integrity in adults. Ann. Neurol. 2008, 63, 652-657. [CrossRef]

78. Bętkowska-Korpała, B. Dynamics of neuroticism in the healing process of individuals addicted to alcohol. Health Psychol. Rep. 2015, 3, 69-84. [CrossRef]

79. Bottlender, M.; Soyka, M. Impact of different personality dimensions (NEO Five-Factor Inventory) on the outcome of alcohol-dependent patients 6 and 12 months after treatment. Psychiatry Res. 2005, 136, 61-67. [CrossRef] [PubMed]

80. Sutin, A.R.; Terracciano, A. Personality traits and body mass index: Modifiers and mechanisms. Psychol. Health 2016, 31, 259-275. [CrossRef] [PubMed]

81. Zallar, L.J.; Farokhnia, M.; Tunstall, B.J.; Vendruscolo, L.F.; Leggio, L. The role of the ghrelin system in drug addiction. Int. Rev. Neurobiol. 2017, 136, 89-119. [PubMed]

82. Emery, R.L.; Levine, M.D. Questionnaire and behavioral task measures of impulsivity are differentially associated with body mass index: A comprehensive meta-analysis. Psychol. Bull. 2017, 143, 868-902. [CrossRef] [PubMed] 
83. McClelland, J.; Dalton, B.; Kekic, M.; Bartholdy, S.; Campbell, I.C.; Schmidt, U. A systematic review of temporal discounting in eating disorders and obesity: Behavioural and neuroimaging findings. Neurosci. Biobehav. Rev. 2016, 71, 506-528. [CrossRef]

84. Kramer, R.S.S. Sexual dimorphism of facial width-to-height ratio in human skulls and faces: A meta-analytical approach. Evol. Hum. Behav. 2017, 38, 414-420. [CrossRef]

85. Manning, J.T. Digit Ratio: A Pointer to Fertility, Behavior and Health; Rutgers University Press: New Brunswick, NJ, USA, 2002.

C 2019 by the authors. Licensee MDPI, Basel, Switzerland. This article is an open access article distributed under the terms and conditions of the Creative Commons Attribution (CC BY) license (http://creativecommons.org/licenses/by/4.0/). 